\title{
SYNTHESIS, BIOLOGICAL EVALUATION AND IN SILICO STUDIES OF PYRAZOLINE AND ITS METAL COMPLEXES AS ANTI-AMOEBIC AGENTS
}

\author{
I. Irfan ${ }^{1,2}$, M. Irfan', M. Abid ${ }^{2, *}$ and A. Azam ${ }^{1, *}$ \\ ${ }^{1}$ Department of Chemistry, Jamia Millia Islamia, Jamia Nagar, New Delhi, India \\ ${ }^{2}$ Department of Biosciences, Jamia Millia Islamia, Jamia Nagar, New Delhi, India \\ *E-mail : amir_sumbul@yahoo.co.in,mabid@jmi.ac.in
}

\begin{abstract}
A series of pyrazolines and metal complexes of 3-(furyl)-2-pyrazoline were design, synthesized and their structures were provided by FT-IR, ${ }^{1} \mathrm{H}-\mathrm{NMR},{ }^{13} \mathrm{C}-\mathrm{NMR}$, ESI-MS and elemental analysis. These compounds were assessed for their in-vitro anti-amoebic activity against HMI:IMSS strain of Entamoeba histolytica and compared with standard drug metronidazole. Pyrazolines showed better activity in comparison to its mannich base, but the activity of metal complexes is much more promising than pyrazolines. The docking and ADMET studies were also conducted to investigate the probable mode of action. From the docking studies, it showed that E. histolytica thioredoxin reductase protein showed an active site for binding affinity.

Keywords: Pyrazoline, Metal complexes, E. histolytica, Docking and ADMET studies.
\end{abstract}

(C) RASĀYAN. All rights reserved

\section{INTRODUCTION}

Amoebiasis is an infection of the human gut caused by the protozoan parasite E. histolytica. More than 50 million people are affected per year. ${ }^{1}$ Infection from this parasite affects the gastrointestinal tract, liver abscesses, destruction of tissue and produce amoebic colitis. ${ }^{2}$ Metronidazole has been considered as the drug of choice against amoebic dysentery. However, this drug shows serious side effects such as genotoxicity, carcinogenicity and hematuria. ${ }^{3-7}$ Moreover, resistance from the standard drug metronidazole increased. ${ }^{8,9}$ In an effort to improve therapy for amoebic dysentery, the creation of chemical libraries is necessary to obtain novel drugs with high activity. The compound which has pyrazoline ring demonstrated good activities including antibacterial, ${ }^{10}$ antifungal, ${ }^{11}$ and antimicrobial. ${ }^{12}$ Recent years have witnessed an increasing interest in metal complexes derived from pyrazoline due to their use as tools for studying the biological activity and emerging therapeutic potentials for a variety of disease. Our continuing interest in anti-amoebic drugs and the desire to explore such types of compounds and biological importance of pyrazolines encourage us to develope new pyrazoline and to enhance their efficacy by introducing metal in their molecular structure. In a recent year from our lab, we have reported several compounds that showed very promising $\mathrm{IC}_{50}$ value, some of them are shown in the (Fig.-1). I $\left(\mathrm{IC}_{50}=0.12 \mu \mathrm{M}\right), \mathbf{I I}\left(\mathrm{IC}_{50}=0.47 \mu \mathrm{M}\right), \mathbf{I I I}\left(\mathrm{IC}_{50}=0.37 \mu \mathrm{M}\right)$ and $\mathbf{I V}\left(\mathrm{IC}_{50}=0.38 \mu \mathrm{M}\right)^{.13-17}$ These results give us a designing strategy. Herein, we have synthesized Pyrazoline derivatives and metal complexes of 3-(furyl)2-pyrazoline. To find the promising target for the treatment of amoebiasis, we have done the molecular docking. It catalyzes the reversible transfer of reducing equivalent between NADPH and thioredoxin. ${ }^{18}$ We have found that thioredoxin reductase is a target for nitroimidazole based drugs in E.histolytica. ${ }^{19}$

\section{Chemistry}

\section{EXPERIMENTAL}

Pyrazoline derivatives were synthesized from Mannich base precursor. The Mannich base precursor (1-6) was prepared by the reaction of ketone, formaldehyde and dimethylamine hydrochloride as shown in

Rasayan J. Chem., 12(4), 2082-2096(2019)

http://dx.doi.org/10.31788/RJC.2019.1245320

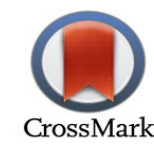


(Scheme-1). ${ }^{20}$ The reaction is effected from amount of $\mathrm{HCl}$ and solvent. The 2-acetyl furan ketone, 2acetyl thiophene ketone and methyl phenyl ketone give high yield above $72 \%$ in comparison to the substituted methyl phenyl ketone.

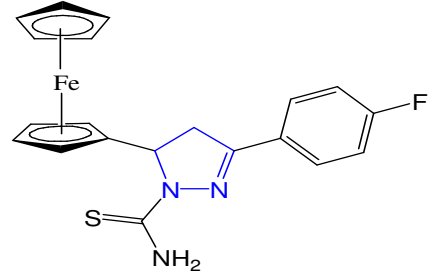

(i)

$\mathrm{IC}_{50}=0.12 \mu \mathrm{M}$

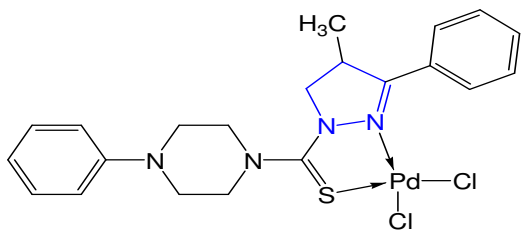

$\mathrm{IC}_{50}=0.37 \mu \mathrm{M}$

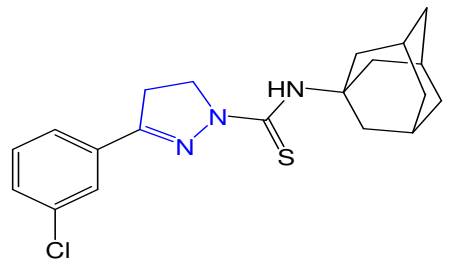

$\mathrm{IC}_{50}=0.47 \mu \mathrm{M}$

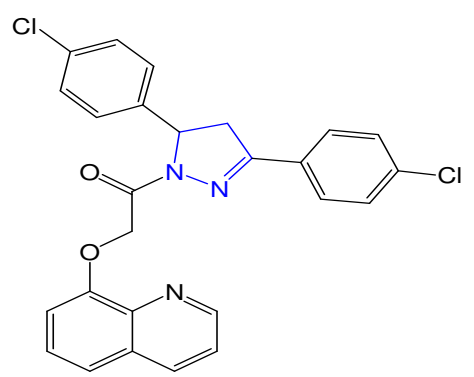

(iv)

Fig.- 1: Some Previously Reported Pyrazoline including Metal Complexes showing Potent Antiamoebic Activity ${ }^{15-18}$

But the yield of Mannich base of propiophenone and substituted propiophenone (14-16) formed by the previous method was very low but it gave satisfying yield about $91 \%$ by mixing propiophenone and substituted propiophenone with paraformaldehyde in the mixture of ethanol and conc. $\mathrm{HCl}^{21}$ Pyrazolines were synthesised by the reaction of Mannich base with $85 \%$ hydrazine in the saturated aqueous sodium bicarbonate solution, one equivalent of hydrazine reaction with two equivalent of Mannich base to form the pyrazoline (7-12,17-19). ${ }^{22}$ The pyrazoline (13) was prepared by reaction of Mannich base in methanol with hydrazine hydrate and sodium hydroxide. The preparation of metal complexes is given in the (Scheme-3).

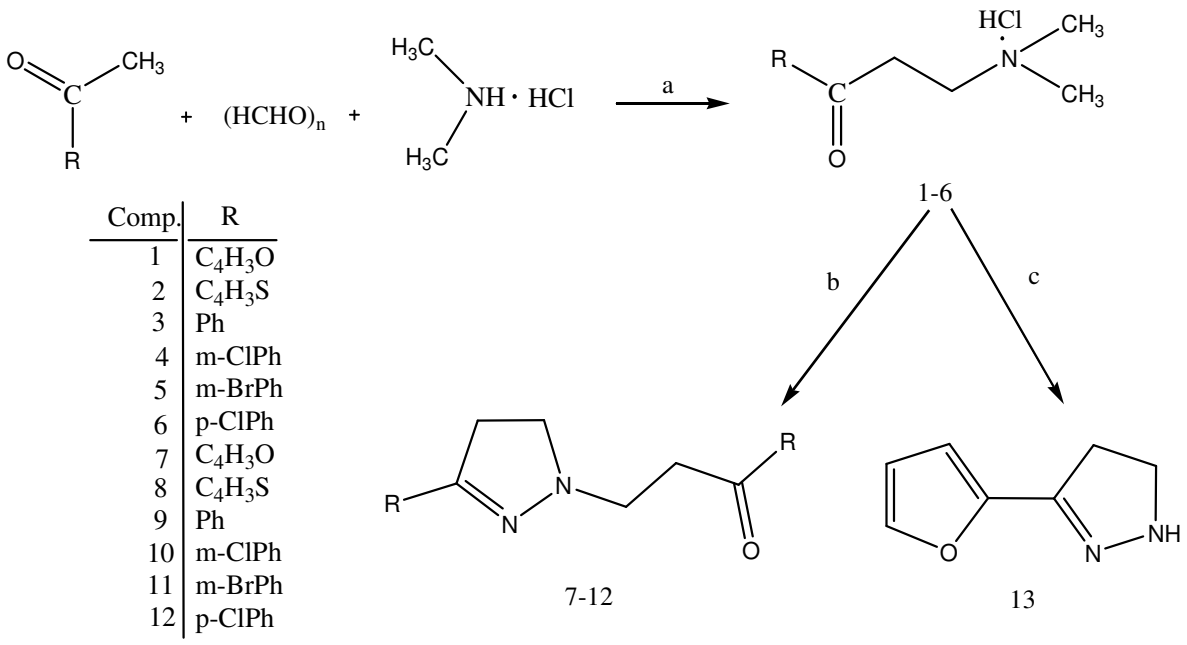

Scheme- 1: The Synthesis of Pyrazoline Derivatives (7-12) and 13. (a) con HCl, acetone, Reflux, 2 h; (b) Hydrazine Hydrate, aq $\mathrm{NaHCO}_{3}, 45$ min; (c) Hydrazine Hydrate, MeOH, 45 min. 
RASĀYAN J. Chem.

Vol. 12 | No. 4 |2082 - 2096| October - December | 2019

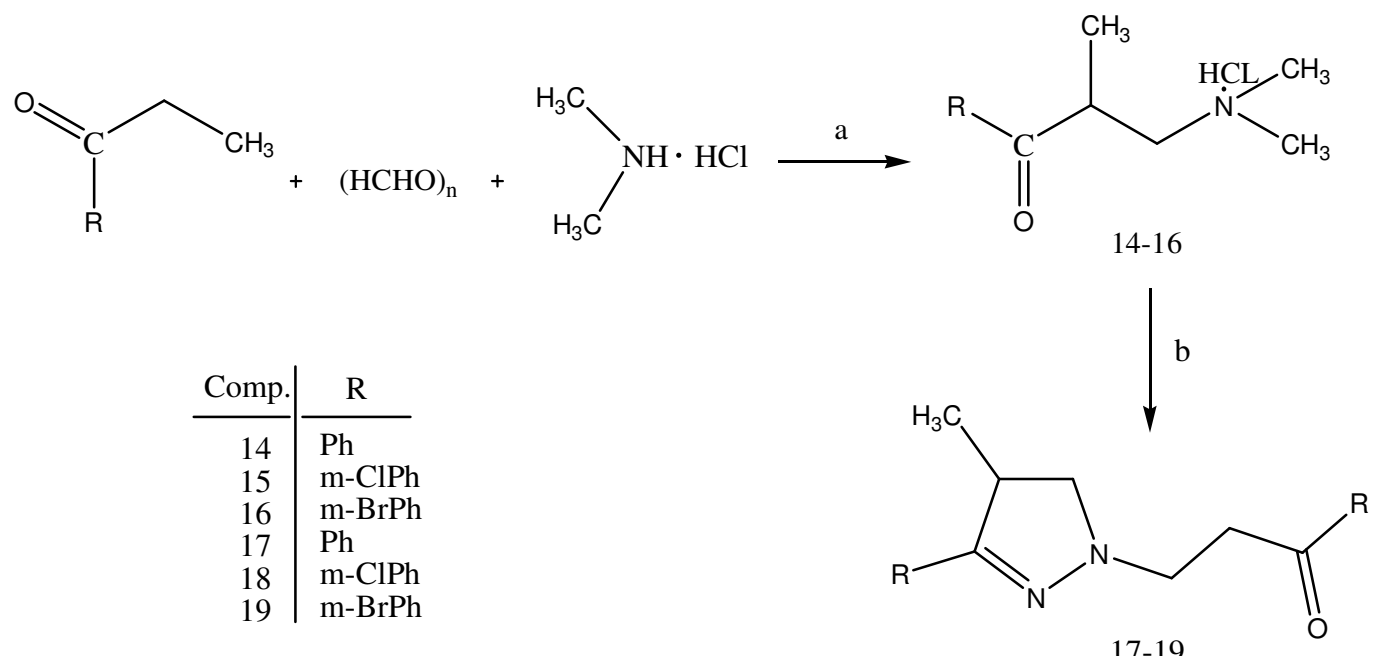

Scheme - 2: The Synthesis of Pyrazoline Derivatives (17-19). (a) Conc HCl, EtOH, Reflux, 2 h; (b) Hydrazine Hydrate, aq $\mathrm{NaHCO}_{3}, 45 \mathrm{~m}$.

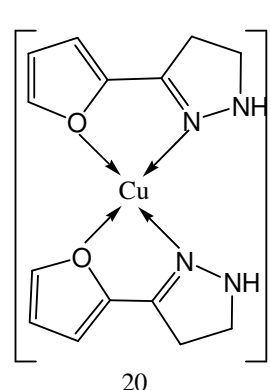

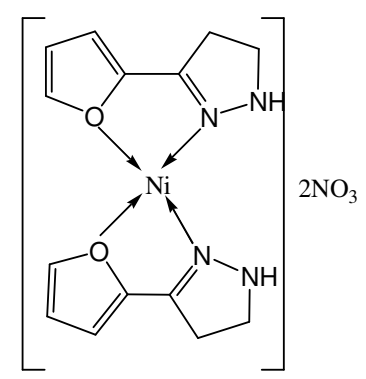

21
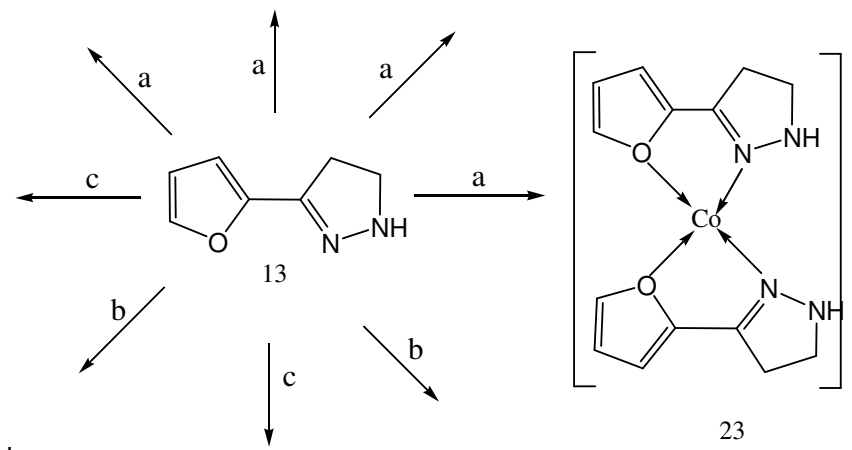

23

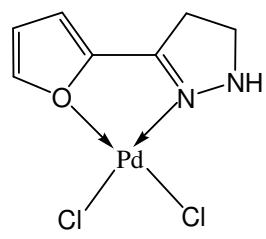

24

Scheme- 3: The Synthesis of Pyrazoline Metal Complexes (20-27). (a) EtOH, Reflux, 2h; (b) MeOH, Reflux, 8h;

(c) $\mathrm{MeOH}$, Reflux, $4 \mathrm{~h}$.

The metal complexes were also found to be stable at room temperature. Metal complexes is soluble in DMF and DMSO. The elemental analysis confirmed that the Pd, Pt, Ru, and $\mathrm{Fe}$ complexes have 1:1 and $\mathrm{Co}, \mathrm{Cu}, \mathrm{Ni}$ complexes have 1:2 molar ratio. In the IR characteristic band of $v(\mathrm{C}-\mathrm{N})$ and $v($ aliph-C-H) in the Mannich base showed at 1213-1257 $\mathrm{cm}^{-1}$ and 2925-2959 $\mathrm{cm}^{-1}$ respectively. Due to the ring closer pyrazoline showed $v(\mathrm{C}=\mathrm{N})$ stretch at $1516-1167 \mathrm{~cm}^{-1}$. Absorption bands at $1144-1167 \mathrm{~cm}^{-1}$ were due to 
the $v(\mathrm{C}-\mathrm{N})$ stretch vibrations, which also showed the formation of the desire compounds. The $v(\mathrm{C}=\mathrm{O})$ band was in the range of $1634-1678 \mathrm{~cm}^{-1}$ and absorption bands at $3126 \mathrm{~cm}^{-1}$ is due to vibration modes of $\mathrm{N}-\mathrm{H}$ functions. The bands at $1550 \mathrm{~cm}^{-1}$ for azomethine $(\mathrm{C}=\mathrm{N})$, shifts during complex formation. This phenomenon may indicate that the nitrogen atom coordinates the metal ion. ${ }^{23}$ The strong bands at 3126 $\mathrm{cm}^{-1}$ for N-H which remains unchanged during complex formation. This shows that the NH group is not involved in the complex formation. The characteristic bands of the furan ring of the free ligand upon complex formation shift respectively. This confirmed the coordination of metal ions by the heterocyclic oxygen atom. ${ }^{24}$ The new bands at about $523-563 \mathrm{~cm}^{-1}, 430-490 \mathrm{~cm}^{-1}$ may correspond to the $\mathrm{v}(\mathrm{M}-\mathrm{N})$ and $\mathrm{v}(\mathrm{M}-\mathrm{O})$ involving the $\mathrm{N}$-atom and $\mathrm{O}$-atom of the furan and pyrazoline ring. ${ }^{25}$ The ${ }^{1} \mathrm{H}-\mathrm{NMR}$ study of the pyrazoline further confirm the formation of the compounds. The protons of a pyrazoline (7-12) at $\mathrm{C}_{4}$ and $\mathrm{C}_{5}$ carbons appeared as broad triplet in the range 3.16-4.67 $(\mathrm{J}=7.1-7.9 \mathrm{~Hz})$ and $3.51-7.31(\mathrm{~J}=6.8-8.9) \mathrm{ppm}$ respectively (Fig.-2). In the pyrazoline (17-19) the $\mathrm{H}_{\mathrm{c}}$ protons at $\mathrm{C}_{4}$ carbon appeared down field in the region 2.86-3.91 as multiplate in all the compounds and the germinal protons $\mathrm{H}_{\mathrm{a}}$ and $\mathrm{H}_{\mathrm{b}}$ at C-5 carbon exhibited two signals. The $\mathrm{CH}_{3}$ proton attached to the pyrazoline nucleus at $\mathrm{C}-4$ appeared as a doublet in $\delta 1.0-1.38(\mathrm{~J}=6.6-6.4 \mathrm{~Hz}) \mathrm{ppm}$ region. The 3-furyl-2-pyrazoline (13) shows a singlet at 7.24 due to $\mathrm{N}-\mathrm{H}$ proton. The structure of the pyrazolines were further supported by ${ }^{13} \mathrm{C}-\mathrm{NMR}$, the $\mathrm{C}_{4}$ and $\mathrm{C}_{5}$ carbon of the cyclized pyrazoline ring in compound (7-12) resonate at 43.5-78.5 and 40.2-58.6 ppm and compound (1719) showed at 38.3-45.3 and 54.7-56.1 ppm.

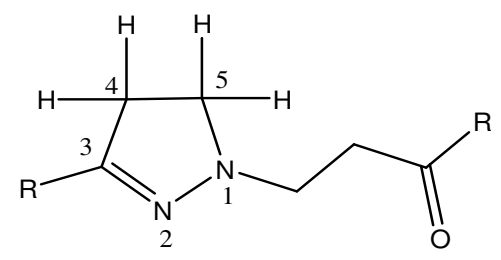

7-12

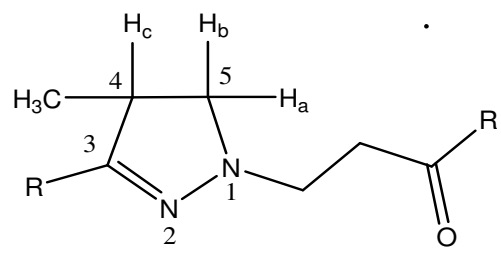

$17-19$

Fig.- 2: Protons of Pyrazoline Derivatives

All the compounds showed a signal at 142.2-164.3 ppm assigned to azomethine carbon of pyrazoline ring. The signals for $(\mathrm{C}=\mathrm{O})$ was in the region of 147.87-245.2 ppm. The $\mathrm{CH}_{3}$ group of pyrazoline ring (17-19) showed signal at 13.8-16.9 ppm. The 3-furyl-2-pyrazoline (13) showed a signal at $152.63 \mathrm{ppm}$ due to $\mathrm{C}=\mathrm{N}$ carbon. The ${ }^{1} \mathrm{H}-\mathrm{NMR}$ spectra did not show significant differences between the ligand (3furyl-2-pyrazoline) and their complexes. The electronic spectra exhibited three absorption bands at 287$299 \mathrm{~cm}^{-1}, 231-241 \mathrm{~cm}^{-1}$ and $214-228 \mathrm{~cm}^{-1}$ assignable to $\mathrm{n} \rightarrow \sigma^{*}, \mathrm{n} \rightarrow \pi^{*}$ and $\pi \rightarrow \pi *$ transition, respectively. The band at $287-299 \mathrm{~cm}^{-1}$ assigned to the $\mathrm{n} \rightarrow \sigma^{*}$ transition of the azomethane nitrogen, the absorption band at $231-241 \mathrm{~cm}^{-1}$ is because of the $\mathrm{n} \rightarrow \pi^{*}$ transition due to the presence of carbonyl oxygen of $\mathrm{C}=\mathrm{O}$ group and the absorption band at $214-228 \mathrm{~cm}^{-1}$ due to the $\pi \rightarrow \pi *$ transition of the phenyl ring. The absorption band at $227 \mathrm{~cm}^{-1}$ is observed due to the $\pi \rightarrow \pi *$ transition of the furan ring. The UVVIS spectra of the metal complexes studied in the UV region in DMSO in the range 200-800 nm. The UV-VIS spectrum of ligand (13) exhibit two bands at 210 and $315 \mathrm{~nm}$. The band at $210 \mathrm{~nm}$ is assigned to $\pi-\pi^{*}$ transition of furan portion, and the one at $315 \mathrm{~nm}$ is due to $\mathrm{n}-\pi^{*}$ transitions of the azomethine moiety, respectively. However, these absorptions were red shift with respect to ligands depending on the types of the metal ions present. The magnetic moments of 1.7-1.9 B.M. fall within the range normally observed for square planer $\mathrm{Cu}(\mathrm{II})$ complexes and also indicate the presence of one unpaired electron in the complexes. The electronic spectra of $\mathrm{Cu}$ (II) complexes of ligand showed bands at 567-645 $\mathrm{nm}$ suggesting a tetragonal configuration around $\mathrm{Cu}$ (II) ion. These bands are accordingly assigned due to ${ }^{2} \mathrm{~B}_{1 \mathrm{~g}} \rightarrow{ }^{2} \mathrm{~A}_{1 \mathrm{~g}},{ }^{2} \mathrm{~B}_{1 \mathrm{~g}} \rightarrow{ }^{2} \mathrm{~B}_{2 \mathrm{~g}}$ and ${ }^{2} \mathrm{~B}_{1 \mathrm{~g}} \rightarrow{ }^{2} \mathrm{Eg}$ transitions, respectively. The magnetic moment of $\mathrm{Co}$ (II) complexes were found to be 4.47B.M. This value is indicative of an octahedral geometry for Co(II) complexes. ${ }^{27}$ The electronic spectra of Co(II) complexes showed bands at 630 and $412 \mathrm{~nm}$ regions which may be assigned to ${ }^{4} \mathrm{~T}_{1 \mathrm{~g}} \rightarrow{ }^{4} \mathrm{~A}_{2 \mathrm{~g}}(\mathrm{~F})$ and ${ }^{4} \mathrm{~T}_{1 \mathrm{~g}} \rightarrow{ }^{4} \mathrm{~T}_{1 \mathrm{~g}}(\mathrm{P})$ transitions, respectively. The magnetic moment, together with position of the bands indicates the octahedral geometry for Co (II) complexes. ${ }^{28}$ The electronic spectra of the $\mathrm{Ni}$ (II) complexes exhibited two bands at 640 and 410nm. The Ni(II) complexes exhibit magnetic moment 
value of 3.11 B.M., which lie within the range reported for the majority of Ni(II) octahedral complexes. ${ }^{29}$ The magnetic moment for Fe(II) complex was found to be 4.90 B.M. which lies within the range for high spin octahedral Fe(II) complexes. ${ }^{30}$ The diffuse reflectance spectrum showed two bands at 490 and $640 \mathrm{~nm}$ which was assigned to ${ }^{5} \mathrm{~T}_{2 \mathrm{~g}} \rightarrow{ }^{2} \mathrm{E}_{\mathrm{g}}$ and charge transfer transitions, respectively. The magnetic moment value and position of the bands, therefore, confirm the octahedral geometry. The electronic spectra of platinum(II) and palladium(II) complexes are indicative of square planar geometry. The ground state is ${ }^{1} \mathrm{~A}_{1} \mathrm{~g}$ and the excited states are ${ }^{1} \mathrm{~A}_{2} \mathrm{~g},{ }^{1} \mathrm{~B}_{1} \mathrm{~g}$ and ${ }^{1} \mathrm{Eg}$ in order of increasing energy. ${ }^{31-33}$ The ground state of ruthenium(II) in an octahedral environment is ${ }^{1} \mathrm{~A}_{1} \mathrm{~g}$ and only two spin allowed transitions, ${ }^{1} \mathrm{~A}_{1} \mathrm{~g} \rightarrow{ }^{1} \mathrm{~T}_{1} \mathrm{~g}$ and ${ }^{1} \mathrm{~A}_{1} \mathrm{~g} \rightarrow{ }^{1} \mathrm{~T}_{2} \mathrm{~g}$ are expected. ${ }^{34}$
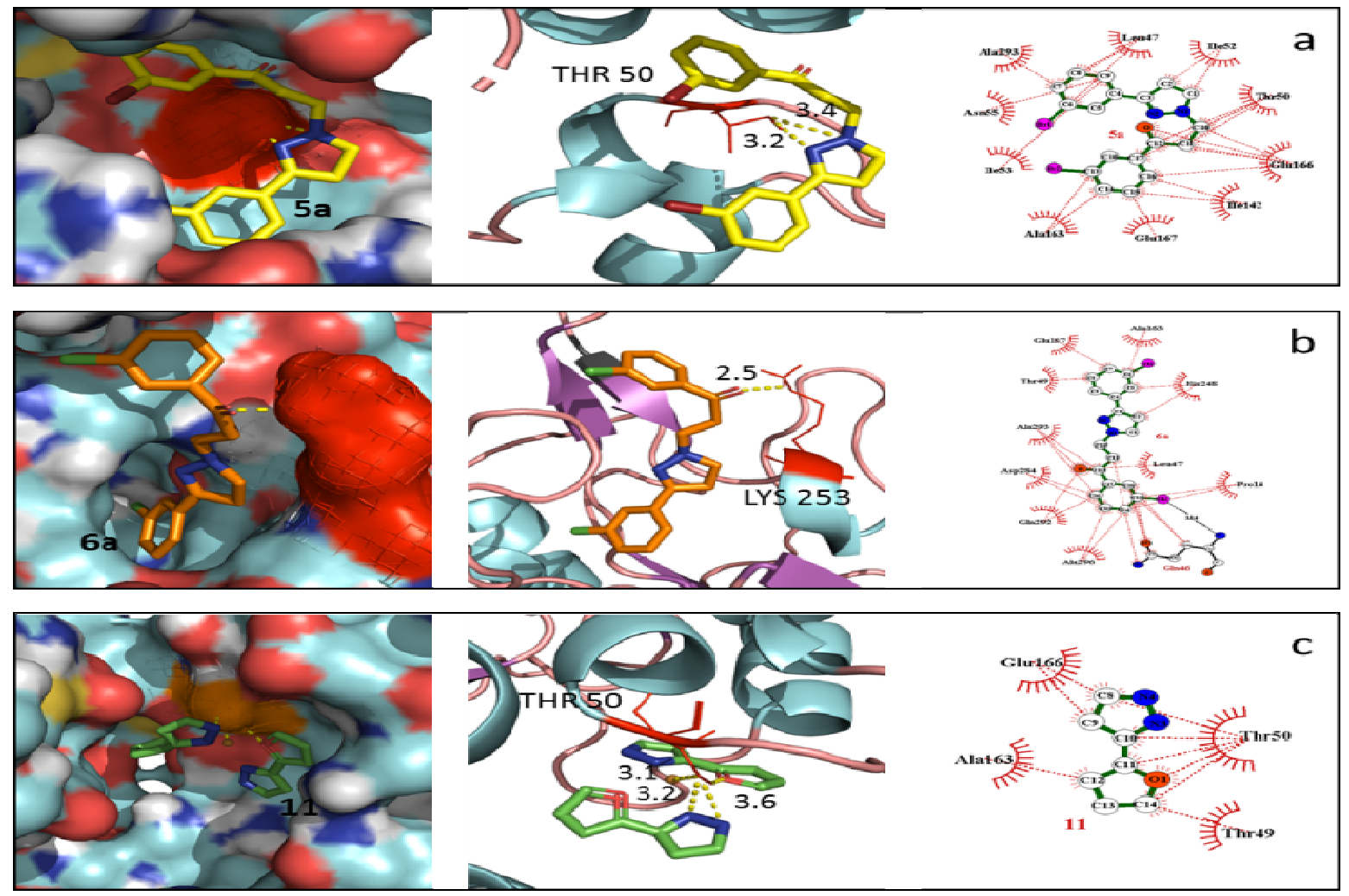

Fig.-3: Molecular Docking of Compounds 11, 12 And 20 With Thioredoxin Reductase of E. Histolytica (Ehtrr). Position of Ligand In Binding Pocket Of Target Protein, Interaction With Residue And Ligplot Images For

\section{Biological Study}

Compounds (a) 11; (b) 12; and (c) 20.

\section{Antiamoebic Activity}

All the Mannich base, pyrazoline derivatives and metal complexes were screened for antiamoebic activity against HMI:IMSS strain of E. histolytica. ${ }^{35}$ Detailed results are given in the (Table-1 and 2). The MNZ was used as the standard drug and had $\mathrm{IC}_{50}=1.8 \mu \mathrm{M}$. The biological data suggested that the Mannich base showed an $\mathrm{IC}_{50}$ value in the range 2.02-10.32 $\mu \mathrm{M}$. Out of nine mannich base, compound 1 with a furan ring substitution, compound 3 with the phenyl ring and compound $\mathbf{4}$ with the chloro-phenyl ring showed low $\mathrm{IC}_{50}$ value $\left(1, \mathrm{IC}_{50}=2.53 \mu \mathrm{M} ; \mathbf{3}, \mathrm{IC}_{50}=2.35 \mu \mathrm{M}\right.$; and4, $\left.\mathrm{IC}_{50}=2.02 \mu \mathrm{M}\right)$ than other Mannich base. The pyrazoline derivative showed better antiamoebic activity as compared to Mannich base. The compound having 3-chloro $\left(\mathbf{1 0}, \mathrm{IC}_{50}=1.5 \mu \mathrm{M}\right)$, 3-Bromo $\left(\mathbf{1 1}, \mathrm{IC}_{50}=0.81 \mu \mathrm{M}\right)$ and 4-Chloro $\left(\mathbf{1 2}, \mathrm{IC}_{50}=0.14 \mu \mathrm{M}\right)$ substitution on the pyrazoline ring were distinctly showed good activity. The biological data showed that the pyrazoline (13) showed an $\mathrm{IC}_{50}=1.92 \mu \mathrm{M}$. The complex formation of Ligand (13) with $\mathrm{Co}(\mathrm{II}), \mathrm{Cu}(\mathrm{II})$, $\mathrm{Ni}(\mathrm{II}), \mathrm{Fe}(\mathrm{II}), \mathrm{Pd}(\mathrm{II}), \mathrm{Pt}(\mathrm{II})$ and $\mathrm{Ru}(\mathrm{II})$ results in complexes which showed $\mathrm{IC}_{50}$ value in the range 0.096$3.14 \mu \mathrm{M}$. Complexes showed better than its ligands in most of the cases. The metal complexes (20) having $\mathrm{IC}_{50}=0.09 \mu \mathrm{M}$, its showed best activity among other compounds. 


\section{In silico Physio-Chemical Properties}

Mostly drugs fail when comes to clinical trial because of their bad physio-chemical properties. This prediction becomes very popular in drug discovery process to screen out the drug-like molecules at initial stage. Here, we did insilico physio-chemical prediction for the compound (1-19). Silico physio-chemical showed that all the compounds have drug-like properties. All compound follow Lipinski's rule of 5. Compound (13) showed best result in comparison to other compound. Results of in silico physiochemical prediction are summarized in (Table-3).

\section{The ADMET Properties}

For the effective drugs interaction between pharmacokinetics, toxicity and potency are crucial. The pharmacokinetic profile of a compound defines its ADME properties. In this paper, we find the pharmoco kinetic properties of compound (1-19). Among all compounds 7, 8, 11 and13 found to be carcinogenic in nature. Absorption and distribution of all the compounds are within range. Mannich base is shown skin sensitivity but its pyrazoline does not show any skin sensitivity. Results of ADMET are summarized in (Table-4).

Table- 1: In Vitro Antiamoebic Activity of Mannich Base and Pyrazolines.

\begin{tabular}{c|c|c}
\hline \multirow{2}{*}{ Compound } & \multicolumn{2}{|c}{ Antiamoebic activity } \\
\cline { 2 - 3 } & $\mathrm{IC}_{50}(\mu \mathrm{M})^{\mathrm{a}}$ & S.D. \\
\hline 1 & 2.35 & 0.04 \\
\hline 2 & 5.01 & 0.05 \\
\hline 3 & 2.35 & 0.05 \\
\hline 4 & 2.02 & 0.03 \\
\hline 5 & 7.69 & 0.02 \\
\hline 6 & 9.07 & 0.05 \\
\hline 7 & 1.06 & 0.03 \\
\hline 8 & 3.77 & 0.03 \\
\hline 9 & 4.01 & 0.02 \\
\hline 10 & 1.05 & 0.03 \\
\hline 11 & 0.18 & 0.01 \\
\hline 12 & 0.14 & 0.02 \\
\hline 13 & 1.92 & 0.02 \\
\hline 14 & 8.46 & 0.04 \\
\hline 15 & 10.32 & 0.01 \\
\hline 16 & 8.11 & 0.01 \\
\hline 17 & 2.13 & 0.01 \\
\hline 18 & 2.24 & 0.04 \\
\hline 19 & 5.21 & 0.02 \\
\hline Metronidazole & 1.8 & 0.01 \\
\hline
\end{tabular}

${ }^{\mathrm{a}}$ Three separate assays done. ${ }^{\mathrm{b}}$ Standard deviation.

Table-2: In vitro Antiamoebic Activity of Metal Complexes.

\begin{tabular}{c|c|c}
\hline \multirow{2}{*}{ Metal complexes } & \multicolumn{2}{|c}{ Antiamoebic activity } \\
\cline { 2 - 3 } & $\mathrm{IC}_{50}(\mu \mathrm{M})^{\mathrm{a}}$ & S.D. $^{\mathrm{b}}$ \\
\hline 20 & 0.09 & 0.01 \\
\hline 21 & 0.36 & 0.04 \\
\hline 22 & 0.31 & 0.01 \\
\hline 23 & 2.5 & 0.02 \\
\hline 24 & 1.49 & 0.01 \\
\hline 25 & 2.17 & 0.03 \\
\hline 26 & 3.14 & 0.02 \\
\hline 27 & 2.81 & 0.01 \\
\hline Metronidazole & 1.8 & 0.01 \\
\hline a Thy
\end{tabular}

\footnotetext{
${ }^{\mathrm{a}}$ Three separate assays done. ${ }^{\mathrm{b}}$ Standard deviation.
} 
Table- 3: Physio-Chemical Parameters of (1-19)

\begin{tabular}{c|c|c|c|c|c|c}
\hline Compound. & Mol. Wt. & LogP & RB & HBA & HBD & $\begin{array}{c}\text { Surface } \\
\text { area }\end{array}$ \\
\hline 1 & 203.66 & 1.8358 & 4 & 3 & 0 & 84.125 \\
\hline 2 & 219.737 & 2.3043 & 4 & 3 & 0 & 88.971 \\
\hline 3 & 213.708 & 2.2428 & 4 & 2 & 0 & 91.331 \\
\hline 4 & 248.153 & 2.8962 & 4 & 2 & 0 & 101.634 \\
\hline 5 & 292.604 & 3.0053 & 4 & 2 & 0 & 105.198 \\
\hline 6 & 248.153 & 2.8962 & 4 & 2 & 0 & 101.634 \\
\hline 7 & 258.277 & 2.555 & 5 & 5 & 0 & 110.048 \\
\hline 8 & 290.413 & 3.4924 & 5 & 5 & 0 & 119.74 \\
\hline 9 & 278.355 & 3.3694 & 5 & 3 & 0 & 124.459 \\
\hline 10 & 347.245 & 4.6762 & 5 & 3 & 0 & 145.066 \\
\hline 11 & 436.147 & 4.8944 & 5 & 3 & 0 & 152.194 \\
\hline 12 & 347.245 & 4.6762 & 5 & 3 & 0 & 145.066 \\
\hline 13 & 136.154 & 0.977 & 1 & 3 & 1 & 58.730 \\
\hline 14 & 227.735 & 2.4888 & 4 & 2 & 0 & 97.696 \\
\hline 15 & 262.18 & 3.1422 & 4 & 2 & 0 & 207.999 \\
\hline 16 & 306.631 & 3.2513 & 4 & 2 & 0 & 111.563 \\
\hline 17 & 292.382 & 3.6154 & 5 & 3 & 0 & 130.824 \\
\hline 18 & 361.277 & 4.9222 & 5 & 3 & 0 & 151.431 \\
\hline 19 & 450.174 & 5.1404 & 5 & 3 & 0 & 158.559 \\
\hline
\end{tabular}

$\mathrm{RB}=$ Rotatable bond, $\mathrm{HBA}=\mathrm{H}$ Accepter, $\mathrm{HBD}=\mathrm{H}$ Donor

\section{Docking Study}

Thioredoxin reductase is a protozoan protein which plays an important role in protozoan's defense mechanism. ${ }^{36}$ Here, we determined the interaction of our lead compounds viz.11, 12 and 20 with thioredoxin reductase of E. histolytica (EhTrR) using AutodockVina 4.2 tool. Compound 11, which showed comparatively less activity among three, showed two interactions with THR 50 residue with bond angle 3.2 and 3.4 A. Similarly, compound 12 showed interaction with LYS 253 residue with a bond angle of $2.5 \AA$. Cu(II) metal complex (20) also bind with THR 50 and showed much interaction between ligand and amino acid residue with bond length of 3.1,3.2 and 3.6 ̊. The binding energy was calculated as -6.7 , -7.7 and $-7.0 \mathrm{Kcal} / \mathrm{mol}$ for the compound 11, 12 and 20, respectively. THR 50 and LYS 253 are important amino acid residues which involve in the FAD binding pocket of target protein $E h T r R .{ }^{37}$ Thus, docking results give significant insights on the possible mechanism action of these compounds. Moreover, in vitro anti-amoebic results were also supported by docking studies.

\section{Chemistry}

\section{RESULTS AND DISCUSSION}

Reactions were monitored by pre-coated aluminum plate silica gel $60 \mathrm{~F}_{254}$ TLC plates. All chemicals and solvents were purchased from Sigma-Aldrich Company. Melting points were recorded on the KSW melting point apparatus. Elemental analysis $(\mathrm{C}, \mathrm{H}, \mathrm{N})$ was carried out on HeraeusVario EL III analyzer by CDRI, Lucknow, India. From Shimadzu UV-1601 PC UV-Visible spectrophotometer electronic spectra were recorded. On Perkin Elmer model 1620 FT-IR spectrophotometer IR was recorded. ${ }^{1} \mathrm{H}-\mathrm{NMR}$ spectra were recorded by Brucker spectroscopic DPX- 300 MHZ spectrometer using Tetramethylsilane (TMS) as an internal standard. Splitting patterns are designated as; s, singlet; d, doublet; m, multiplet and coupling constant $J$ is given in hertz. Chemical shift value is given in (ppm). The FAB mass spectra of all the compounds were recorded by JEOL SX 102/DA-6000 mass spectrometer.

Table- 4: ADMET Properties of Compound (1-19).

\begin{tabular}{|c|c|c|c|c|c|c|}
\hline \multirow[t]{2}{*}{ Comp. } & \multirow{2}{*}{$\begin{array}{c}\text { Water } \\
\text { Solubility }\end{array}$} & \multirow{2}{*}{$\begin{array}{l}\text { HIA } \\
(\%)\end{array}$} & \multicolumn{3}{|c|}{ Permeability } & \multirow{2}{*}{$\begin{array}{l}\text { CYP2D6 } \\
\text { Inhibitor }\end{array}$} \\
\hline & & & Skin & BBB & $\mathrm{CNS}$ & \\
\hline 1 & -1.183 & 94.39 & -2.42 & 0.232 & -3.116 & No \\
\hline
\end{tabular}


RASĀYAN J. Chem.

Vol. 12 | No. 4 |2082 - 2096| October - December | 2019

\begin{tabular}{c|c|c|c|c|c|c}
\hline 2 & -1.975 & 91.99 & -1.833 & 0.319 & -1.908 & No \\
\hline 3 & -1.924 & 93.52 & -1.821 & 0.372 & -1.732 & No \\
\hline 4 & -2.799 & 92.78 & -1.842 & 0.373 & -1.798 & No \\
\hline 5 & -2.945 & 92.714 & -1.844 & 0.356 & -1.798 & Yes \\
\hline 6 & -2.731 & 92.136 & -1.837 & 0.364 & -1.854 & Yes \\
\hline 7 & -3.051 & 97.333 & -2.849 & 0.029 & -2.834 & No \\
\hline 8 & -4.104 & 90.881 & -2.206 & 0.327 & -1.829 & No \\
\hline 9 & -4.049 & 94.101 & -2.218 & 0.399 & -1.348 & No \\
\hline 10 & -5.591 & 92.615 & -2.34 & 0.309 & -1.366 & No \\
\hline 11 & -5.831 & 92.48 & -2.359 & 0.306 & -1.35 & No \\
\hline 12 & -5.457 & 91.79 & -2.341 & 0.312 & -1.31 & No \\
\hline 13 & -1.23 & 93.952 & -2.877 & 0.223 & -3.162 & No \\
\hline 14 & -2.229 & 93.46 & -1.834 & 0.368 & -1.778 & Yes \\
\hline 15 & -3.109 & 92.722 & -1.868 & 0.369 & -1.844 & No \\
\hline 16 & -3.255 & 92.655 & -1.872 & 0.352 & -1.844 & No \\
\hline 17 & -4.454 & 94.518 & -2.261 & 0.331 & -1.328 & No \\
\hline 18 & -5.948 & 93.032 & -2.388 & 0.258 & -1.328 & No \\
\hline 19 & -6.179 & 92.89 & -2.406 & 0.255 & -1.328 & No \\
\hline
\end{tabular}

Table-4: Part -2

\begin{tabular}{|c|c|c|c|c|c|c|c|c|}
\hline \multirow[t]{3}{*}{ Comp. } & \multirow{2}{*}{$\begin{array}{l}\text { CYP2C9 } \\
\text { Inhibitor }\end{array}$} & \multirow{2}{*}{$\begin{array}{c}\text { CYP2C19 } \\
\text { Inhibitor }\end{array}$} & \multirow{2}{*}{$\begin{array}{c}\text { Total } \\
\text { Clearance }\end{array}$} & \multirow{2}{*}{$\begin{array}{c}\text { Renal } \\
\text { OCT2 } \\
\text { Substrate }\end{array}$} & \multirow{2}{*}{$\begin{array}{l}\text { Hepa } \\
\text { Toxicity }\end{array}$} & \multirow{2}{*}{$\begin{array}{c}\text { Skin } \\
\text { Sensitization }\end{array}$} & \multicolumn{2}{|c|}{ Carcinogenicity } \\
\hline & & & & & & & Sum & $\begin{array}{c}\mathrm{XG} \\
\text { Boost }\end{array}$ \\
\hline & No & No & 1.398 & No & No & Yes & No & No \\
\hline 1 & No & No & 1.535 & No & No & Yes & No & No \\
\hline 2 & No & No & 1.308 & Yes & Yes & Yes & No & No \\
\hline 3 & No & No & 1.24 & No & Yes & Yes & No & No \\
\hline 4 & No & No & 1.189 & No & Yes & Yes & No & No \\
\hline 5 & No & No & 1.345 & Yes & Yes & Yes & No & No \\
\hline 6 & No & No & 0.717 & No & No & No & No & No \\
\hline 7 & No & Yes & 0.457 & Yes & No & No & No & No \\
\hline 8 & No & Yes & 0.508 & No & Yes & No & No & No \\
\hline 9 & Yes & Yes & 0.237 & Yes & No & No & No & No \\
\hline 10 & Yes & Yes & 0.193 & Yes & No & No & No & Yes \\
\hline 11 & Yes & Yes & 0.113 & Yes & No & No & No & No \\
\hline 12 & No & No & 0.648 & No & No & Yes & Yes & Yes \\
\hline 13 & No & No & 1.065 & Yes & Yes & Yes & No & No \\
\hline 14 & No & No & 0.997 & No & Yes & Yes & Yes & Yes \\
\hline 15 & No & No & 0.945 & Yes & Yes & Yes & Yes & Yes \\
\hline 16 & Yes & Yes & 0.488 & Yes & Yes & No & No & No \\
\hline 17 & Yes & Yes & 0.231 & Yes & No & No & No & No \\
\hline 18 & Yes & Yes & 0.186 & Yes & No & No & No & No \\
\hline
\end{tabular}

General procedure for the synthesis of Mannich Base (1-6)

A ketone $(0.2 \mathrm{~mol})$, paraformaldehyde $(0.26 \mathrm{~mol}$.) and dimethylamine hydrochloride $(0.26 \mathrm{~mol})$ in $35 \mathrm{ml}$ of ethanol and $0.5 \mathrm{ml}$ of conc. $\mathrm{HCl}$, refluxed about $2 \mathrm{hr}$. After cooling, acetone about $200 \mathrm{ml}$ was added. The crystal formed, washed with acetone and dried in vacuo.

\section{2-Acetylfuran Mannich Base (1)}

White Solid; Yield: 83\%; m.p: $125^{\circ} \mathrm{C}$; IR: $v_{\max } \mathrm{cm}^{-1}$ : 1659 (C=O), 1257 (C-N), 2925 (Aliph-CH), 1257 (CO-C); ${ }^{1} \mathrm{H} \mathrm{NMR}\left(\mathrm{CDCl}_{3}\right)(\delta, \mathrm{ppm}): 2.86\left(\mathrm{t}, 2 \mathrm{H}, \mathrm{CH}_{2}\right), 2.94\left(\mathrm{t}, 2 \mathrm{H}, \mathrm{CH}_{2}\right), 2.36\left(\mathrm{~s}, 6 \mathrm{H}, \mathrm{CH}_{3}\right), 6.92(\mathrm{~d}, 1 \mathrm{H}$, $J=3.4, \mathrm{Ar}-\mathrm{H}), 7.71(\mathrm{~d}, 1 \mathrm{H}, J=1.6, \mathrm{Ar}-\mathrm{H}), 6.64(\mathrm{dd}, 1 \mathrm{H}, J=3.4,1.6, \mathrm{Ar}-\mathrm{H})$.

2-Acetylthiophene Mannich Base (2)

White Solid; Yield: 72\%; m.p: $180^{\circ} \mathrm{C}$; IR: $v_{\max } \mathrm{cm}^{-1}: 1742(\mathrm{C}=\mathrm{O}), 1235(\mathrm{C}-\mathrm{N}), 1227$ (Aliph-CH), 853 (C- 
O-C); ${ }^{1} \mathrm{H}$ NMR $\left(\mathrm{CDCl}_{3}\right)(\delta, \mathrm{ppm}): 2.84\left(\mathrm{t}, 2 \mathrm{H}, \mathrm{CH}_{2}\right), 2.93\left(\mathrm{t}, 2 \mathrm{H}, \mathrm{CH}_{2}\right), 2.33(\mathrm{~s}, 6 \mathrm{H}, \mathrm{Ar}-\mathrm{H}), 6.98(\mathrm{~d}, 1 \mathrm{H}$, $J=3.5, \mathrm{Ar}-\mathrm{H}), 7.48(\mathrm{~d}, 1 \mathrm{H}, J=1.5, \mathrm{Ar}-\mathrm{H}), 6.52(\mathrm{dd}, 1 \mathrm{H}, J=3.5,1.5, \mathrm{Ar}-\mathrm{H})$.

AcetophenoneMannich Base (3)

White Solid; Yield: 88\%; m.p: $88^{\circ} \mathrm{C}$; IR: $v_{\max } \mathrm{cm}^{-1}: 1679(\mathrm{C}=\mathrm{O}), 1226(\mathrm{C}-\mathrm{N}), 2953$ (Aliph-CH), 1466 (Ar $\mathrm{C}=\mathrm{C}) ;{ }^{1} \mathrm{H}$ NMR $\left(\mathrm{CDCl}_{3}\right)(\delta, \mathrm{ppm}): 3.31\left(\mathrm{t}, 2 \mathrm{H}, \mathrm{CH}_{2}\right), 2.99\left(\mathrm{t}, 2 \mathrm{H}, \mathrm{CH}_{2}\right), 2.55\left(\mathrm{~s}, 6 \mathrm{H}, \mathrm{CH}_{3}\right), 7.22-7.86(\mathrm{dd}$, $1 \mathrm{H}, J=3.4,1.6, \mathrm{Ar}-\mathrm{H})$.

3-chloro acetophenoneMannich Base (4)

White Solid; Yield: $42 \%$; m.p: $189^{\circ} \mathrm{C}$; IR: $v_{\max } \mathrm{cm}^{-1}$ : 1689 (C=O), 1213 (C-N), 2967 (Aliph-CH), 1484 (Ar $\mathrm{C}=\mathrm{C}) ;{ }^{1} \mathrm{H}$ NMR $\left(\mathrm{CDCl}_{3}\right)(\delta, \mathrm{ppm}): 3.33\left(\mathrm{t}, 2 \mathrm{H}, \mathrm{CH}_{2}\right), 2.94\left(\mathrm{t}, 2 \mathrm{H}, \mathrm{CH}_{2}\right), 2.71\left(\mathrm{~s}, 6 \mathrm{H}, \mathrm{CH}_{3}\right), 7.04(\mathrm{~d}, 1 \mathrm{H}$, $J=7.4 \mathrm{~Hz}, \mathrm{Ar}-\mathrm{H}), 7.12$ (d, 1H, J=7.4Hz, Ar-H), 7.16 (dd, 1H, Ar-H), 7.09 (s, 1H, Ar-H).

\section{3-bromo acetophenoneMannich Base (5)}

White Solid; Yield: 56\%; m.p: $178^{\circ} \mathrm{C}$; IR: $v_{\max } \mathrm{cm}^{-1}: 1687(\mathrm{C}=\mathrm{O}), 1214$ (C-N), 2969 (Alip-CH), 1473 ( arom $\mathrm{C}=\mathrm{C}) ;{ }^{1} \mathrm{H}$ NMR $\left(\mathrm{CDCl}_{3}\right)(\delta, \mathrm{ppm}): 3.32\left(\mathrm{t}, 2 \mathrm{H}, \mathrm{CH}_{2}\right), 2.94\left(\mathrm{t}, 2 \mathrm{H}, \mathrm{CH}_{2}\right), 2.66\left(\mathrm{~s}, 6 \mathrm{H}, \mathrm{CH}_{3}\right), 7.20(\mathrm{~d}$, $1 \mathrm{H}, J=7.3 \mathrm{~Hz}, \mathrm{Ar}-\mathrm{H}), 7.16(\mathrm{~d}, 1 \mathrm{H}, J=7.3 \mathrm{~Hz}, \mathrm{Ar}-\mathrm{H}), 7.18$ (dd, 1H, Ar-H), 7.14 (s, 1H, Ar-H).

\section{4-Chloro acetophenoneMannich Base (6)}

White Solid; Yield: 62\%; m.p: $194^{\circ} \mathrm{C}$; IR: $v_{\max } \mathrm{cm}^{-1}$ : $1682(\mathrm{C}=\mathrm{O}), 1211(\mathrm{C}-\mathrm{N}), 2966$ (Aliph-CH), 1488 ( arom $\mathrm{C}=\mathrm{C}) ;{ }^{1} \mathrm{H}$ NMR $\left(\mathrm{CDCl}_{3}\right)$ : $(\delta, \mathrm{ppm}): 3.28\left(\mathrm{t}, 2 \mathrm{H}, \mathrm{CH}_{2}\right), 2.81\left(\mathrm{t}, 2 \mathrm{H}, \mathrm{CH}_{2}\right), 2.71\left(\mathrm{~s}, 6 \mathrm{H}, \mathrm{CH}_{3}\right), 7.13(\mathrm{~d}$, $2 \mathrm{H}, J=7.2 \mathrm{~Hz}, \mathrm{Ar}-\mathrm{H}), 7.16(\mathrm{~d}, 2 \mathrm{H}, J=7.2 \mathrm{~Hz}, \mathrm{Ar}-\mathrm{H})$.

\section{General Procedure for the Synthesis Of Mannich Base (14-16)}

For $2 \mathrm{~h}$ reflux suspension of substituted propiophenone $(0.2 \mathrm{~mol})$, paraformaldehyde $(0.26 \mathrm{~mol})$ and dimethylamine hydrochloride $(0.26 \mathrm{~mol})$, used ethanol as a solvent and add $0.5 \mathrm{ml}$ of conc. HCl. After removing the solvent, add a few drops of $\mathrm{HCl}$, and the mixture was extracted with dichloromethane and water. We get a colorless oil as a product.

\section{Propiophenonemannichbase (14)}

Colorless oil; Yield: 91\%; IR: $v_{\max } \mathrm{cm}^{-1}: 1686(\mathrm{C}=\mathrm{O}), 1221(\mathrm{C}-\mathrm{N}), 2950$ (Alip-CH), 1481 (arom C=C); ${ }^{1} \mathrm{H}$ $\operatorname{NMR}\left(\mathrm{CDCl}_{3}\right)(\delta, \mathrm{ppm}): 2.18\left(\mathrm{~s}, 6 \mathrm{H}, \mathrm{CH}_{3}\right), 2.74-2.55\left(\mathrm{dd}, J=5.6,10.3 \mathrm{~Hz}, \mathrm{CH}_{2}\right), 1.16\left(\mathrm{~d}, 3 \mathrm{H}, J=7.04 \mathrm{~Hz}, \mathrm{CH}_{3}\right)$, $3.63(\mathrm{H}, \mathrm{m}, \mathrm{CH}), 7.33-7.45(\mathrm{~m}, 5 \mathrm{H}, \mathrm{Ar}-\mathrm{H})$.

\section{3'-chloropropiophenone Mannich Base (15)}

Colorless oil; Yield: 71\%; IR: $v_{\max } \mathrm{cm}^{-1}: 1676(\mathrm{C}=\mathrm{O}), 1211(\mathrm{C}-\mathrm{N}), 2932\left(\right.$ Alip-CH), 1465 (arom C=C); ${ }^{1} \mathrm{H}$ $\operatorname{NMR}\left(\mathrm{CDCl}_{3}\right)(\delta, \mathrm{ppm}): 2.14\left(\mathrm{~s}, 6 \mathrm{H}, \mathrm{CH}_{3}\right), 2.79-2.32\left(\mathrm{dd}, J=5.6,10.3 \mathrm{~Hz}, \mathrm{CH}_{2}\right), 1.19\left(\mathrm{~d}, 3 \mathrm{H}, J=7.04 \mathrm{~Hz}, \mathrm{CH}_{3}\right)$, $3.68(\mathrm{H}, \mathrm{m}, \mathrm{CH}), 7.11(\mathrm{~d}, 1 \mathrm{H}, J=7.3 \mathrm{~Hz}, \mathrm{Ar}-\mathrm{H}), 7.18(\mathrm{~d}, 1 \mathrm{H}, J=7.3 \mathrm{~Hz}, \mathrm{Ar}-\mathrm{H}), 7.15(\mathrm{dd}, 1 \mathrm{H}, \mathrm{Ar}-\mathrm{H}), 7.04(\mathrm{~s}, 1 \mathrm{H}, \mathrm{Ar}-$ $\mathrm{H})$.

\section{3'-bromopropiophenone Mannich Base (16)}

Colorless oil; Yield: 65\%; IR: $v_{\max } \mathrm{cm}^{-1}: 1678(\mathrm{C}=\mathrm{O}), 1223(\mathrm{C}-\mathrm{N}), 2933$ (Alip-CH), 1468 (arom C=C); ${ }^{1} \mathrm{H}$ $\operatorname{NMR}\left(\mathrm{CDCl}_{3}\right)(\delta, \mathrm{ppm}): 2.16\left(\mathrm{~s}, 6 \mathrm{H}, \mathrm{CH}_{3}\right), 2.72-2.41\left(\mathrm{dd}, J=5.4,10.2 \mathrm{~Hz}, \mathrm{CH}_{2}\right), 1.19\left(\mathrm{~d}, 3 \mathrm{H}, J=7.03 \mathrm{~Hz}, \mathrm{CH}_{3}\right)$, $3.59(\mathrm{H}, \mathrm{m}, \mathrm{CH}), 7.16(\mathrm{~d}, 1 \mathrm{H}, J=7.3 \mathrm{~Hz}, \mathrm{Ar}-\mathrm{H}), 7.12(\mathrm{~d}, 1 \mathrm{H}, J=7.3 \mathrm{~Hz}, \mathrm{Ar}-\mathrm{H}), 7.19(\mathrm{dd}, 1 \mathrm{H}, \mathrm{Ar}-\mathrm{H}), 7.07(\mathrm{~s}, 1 \mathrm{H}, \mathrm{Ar}-$ $\mathrm{H})$.

\section{General Procedure for the Preparation of Pyrazoline Derivatives (7-12,17-19)}

The pyrazoline was prepared by the reaction of the Mannich base $(0.056$ mole $)$ with $85 \%$ hydrazine hydrate $(0.028$ mole $)$ in the aqu sodium bicarbonate and refluxed for $45 \mathrm{~min}$ with staring, After cooling, the reaction mixture was pure in water and the aqueous layer was extracted with dichloromethane. Mixed organic layers, dried over (MgSO4), filtered and concentrated. The compounds were re-crystallized using dichloromethane/ hexane solution $(1: 2)$.

1-(Furan-2-yl)-3-(3-(Furan-2-yl)-4,5-dihydropyrazol-1-yl)propan-1-one (7)

Dirty green solid; Yield: 78\%; m.p. $47^{\circ}$ C; UV: $\lambda_{\max }(\mathrm{nm}): 253,231,291$; IR: $v_{\max } \mathrm{cm}^{-1}: 1247$ (C-O-C), 
1663( $\mathrm{C}=\mathrm{O}), 1550(\mathrm{C}=\mathrm{N}), 1162(\mathrm{C}-\mathrm{N}) ;{ }^{1} \mathrm{HNMR}\left(\mathrm{CDCl}_{3}\right):(\delta, \mathrm{ppm}): 2.95\left(\mathrm{t}, 2 \mathrm{H}, \mathrm{J}=6.2 \mathrm{~Hz}\right.$, Aliph- $\left.\mathrm{CH}_{3}\right)$, $2.91\left(\mathrm{t}, 2 \mathrm{H}, J=6.5 \mathrm{~Hz}\right.$, Aliph- $\left.\mathrm{CH}_{3}\right), 3.28\left(\mathrm{t}, 2 \mathrm{H}, \mathrm{J}=7.2 \mathrm{~Hz}, \mathrm{CH}_{3}\right), 3.71\left(\mathrm{t}, 2 \mathrm{H}, J=6.9 \mathrm{~Hz}, \mathrm{CH}_{3}\right), 6.52(\mathrm{~d}, 1 \mathrm{H}$, $J=3.6 \mathrm{~Hz}, \mathrm{Ar}-\mathrm{H}), 7.2(\mathrm{~d}, 1 \mathrm{H}, J=1.9 \mathrm{~Hz}, \operatorname{Ar}-\mathrm{H}), 6.49$ (dd, $1 \mathrm{H}, \mathrm{J}=3.6 \mathrm{~Hz}, 1.9 \mathrm{~Hz}, \operatorname{Ar}-\mathrm{H}), 6.61$ (d, $1 \mathrm{H}, J=3.1$ $\mathrm{Hz}, \mathrm{Ar}-\mathrm{H}) .7 .11$ ( d, $1 \mathrm{H}, J=1.6 \mathrm{~Hz}, \mathrm{Ar}-\mathrm{H}), 6.53(\mathrm{dd}, 1 \mathrm{H}, \mathrm{J}=3.1 \mathrm{~Hz}, 1.6 \mathrm{~Hz}, \mathrm{Ar}-\mathrm{H}) ;{ }^{13} \mathrm{CNMR}\left(\mathrm{CDCl}_{3}\right):(\delta$, ppm): $192.33(\mathrm{C}=\mathrm{O}), 147.87(\mathrm{C}=\mathrm{N}), 54.96\left(\right.$ Aliph- $\left.\mathrm{CH}_{2}\right), 37.99\left(\right.$ Aliph- $\left.\mathrm{CH}_{2}\right), 43.8\left(\mathrm{CH}_{2}\right), 73.5\left(\mathrm{CH}_{2}\right) ; \mathrm{FAB}$ MAS: $m / z\left(\mathrm{M}^{+}+1\right) 256$ calc.258.1. Anal. Calc. $(\%)$ for $\left(\mathrm{C}_{14} \mathrm{H}_{14} \mathrm{~N}_{2} \mathrm{O}_{3}\right) \mathrm{C}, 65.11 ; \mathrm{H}, 5.46, \mathrm{~N}, 10.85$; Found: C, 65.13; H, 5.23, N,10.8.

\section{3-(4,5-dihydro-3-(thiophene-2-yl)pyrazol-1-yl)-1-(thiophene-2-yl)propan-1-one (8)}

Dirty green solid; Yield: $81 \%$; m.p. $55^{\circ}$ C. Found: C, 57.71; H, 4.83, N, 9.47; UV: $\lambda_{\max }(\mathrm{nm}): 227,293$, 236; IR: $v_{\max } \mathrm{cm}^{-1}: 1648(\mathrm{C}=\mathrm{O}), 1511(\mathrm{C}=\mathrm{N}), 1176(\mathrm{C}-\mathrm{N}) ;{ }^{1} \mathrm{HNMR}\left(\mathrm{CDCl}_{3}\right)(\delta, \mathrm{ppm}): 2.97(\mathrm{t}, 2 \mathrm{H}, J=$ 6.4Hz, Aliph- $\left.\mathrm{CH}_{3}\right), 2.94$ (t, 2H, J=6.7 Hz, Aliph- $\left.\mathrm{CH}_{3}\right), 3.17\left(\mathrm{t}, 2 \mathrm{H}, \mathrm{J}=7.9 \mathrm{~Hz}, \mathrm{CH}_{3}\right), 3.51$ (t , 2H , J=8.1 $\left.\mathrm{Hz}, \mathrm{CH}_{3}\right), 6.54(\mathrm{~d}, 1 \mathrm{H}, J=3.9 \mathrm{~Hz}, \mathrm{Ar}-\mathrm{H}), 7.92(\mathrm{~d}, 1 \mathrm{H}, J=1.7 \mathrm{~Hz}, \mathrm{Ar}-\mathrm{H}), 7.01$ (dd, $1 \mathrm{H}, J=3.9 \mathrm{~Hz}, 1.7 \mathrm{~Hz}$, Ar-H), 6.96 (d, 1H, J=4.1 Hz, Ar-H). $7.42(\mathrm{~d}, 1 \mathrm{H}, J=1.8 \mathrm{~Hz}, \mathrm{Ar}-\mathrm{H}), 6.21$ (dd, $1 \mathrm{H}, J=4.1 \mathrm{~Hz}, 1.8 \mathrm{~Hz}, \mathrm{Ar}-$ $\mathrm{H}) ;{ }^{13} \mathrm{CNMR}\left(\mathrm{CDCl}_{3}\right):(\delta, \mathrm{ppm}): 182.96(\mathrm{C}=\mathrm{O}), 148.33(\mathrm{C}=\mathrm{N}), 54.26\left(\right.$ Aliph- $\left.\mathrm{CH}_{2}\right), 37.12$ (Aliph$\left.\mathrm{CH}_{2}\right)$,49.6 $\left(\mathrm{CH}_{2}\right), 78.5\left(\mathrm{CH}_{2}\right)$; FAB MAS: $m / z \quad\left(\mathrm{M}^{+}+1\right) 290$ calc.290.4. Anal. Calc. $(\%)$ for $\left(\mathrm{C}_{14}\right.$ $\mathrm{H}_{14} \mathrm{~N}_{2} \mathrm{OS}_{2}$ ) C, 57.90; H, 4.86, N, 9.65; Found: C,57, 86; H, 4.8; N, 9.61.

\section{3-(4,5-dihydro-3-phenylpyrazol-1-yl)-1-phenylpropan-1-one (9)}

Pale green solid; Yield: 86.3\%; m.p. $95^{\circ}$ C; UV: $\lambda_{\max }(\mathrm{nm}): 297,234,216$; IR: $v_{\max } \mathrm{cm}^{-1}$ : 1673 ( C=O), 1582 $(\mathrm{C}=\mathrm{N}), 1161(\mathrm{C}-\mathrm{N}), 742,694$ (Benzene); ${ }^{1} \mathrm{HNMR}\left(\mathrm{CDCl}_{3}\right)(\delta, \mathrm{ppm}): 2.61$ (t, 2H , J=6.1 Hz, Aliph-CH$\left.{ }_{3}\right)$, $2.72\left(\mathrm{t}, 2 \mathrm{H}, J=6.5 \mathrm{~Hz}\right.$, Aliph- $\left.\mathrm{CH}_{3}\right), 3.56\left(\mathrm{t}, 2 \mathrm{H}, J=7.9, \mathrm{~Hz} \mathrm{CH}_{3}\right), 4.01\left(\mathrm{t}, 2 \mathrm{H}, J=6.8 \mathrm{~Hz}, \mathrm{CH}_{3}\right), 7.25(\mathrm{~m}$, 5H, Ar-H), $7.98(\mathrm{~m}, 5 \mathrm{H}, \mathrm{Ar}-\mathrm{H}) ;{ }^{13} \mathrm{CNMR}\left(\mathrm{CDCl}_{3}\right):(\delta, \mathrm{ppm}): 216.6(\mathrm{C}=\mathrm{O}), 151.8(\mathrm{C}=\mathrm{N}), 53.4\left(\mathrm{Aliph}-\mathrm{CH}_{2}\right)$, 39.5 (Alip- $\left.\mathrm{CH}_{2}\right), 40.2\left(\mathrm{CH}_{2}\right), 66.7\left(\mathrm{CH}_{2}\right)$; FAB MAS: $m / z \quad\left(\mathrm{M}^{+}+1\right) 277.6$ calc.278.14. Anal. Calc. $(\%)$ for $\left(\mathrm{C}_{18} \mathrm{H}_{18} \mathrm{~N}_{2} \mathrm{O}\right) \mathrm{C}, 77.67$; H, 6.52, N, 10.06; Found: C, 77.64; H, 6.43, N, 9.97.

\section{1-(3-chlorophenyl)-3(3-(3-chlorophenyl)-4,5-dihydropyrazol-1-yl)propan-1-one (10)}

Pale green solid; Yield: $65.8 \%$; m.p. $110^{\circ} \mathrm{C}$; UV: $\lambda_{\max }(\mathrm{nm}): 289,231,217$; IR: $v_{\max } \mathrm{cm}^{-1}: 1671(\mathrm{C}=\mathrm{O}), 1589$ $(\mathrm{C}=\mathrm{N}), 1163(\mathrm{C}-\mathrm{N}), 779,783$ (Benzene); ${ }^{1} \mathrm{HNMR}\left(\mathrm{CDCl}_{3}\right)(\delta, \mathrm{ppm}): 2.74$ (t, 2H, J=6.3 Hz, Alip- $\left.\mathrm{CH}_{3}\right)$, $2.67\left(\mathrm{t}, 2 \mathrm{H}, J=6.8 \mathrm{~Hz}\right.$, Alip- $\left.\mathrm{CH}_{3}\right), 3.46\left(\mathrm{t}, 2 \mathrm{H}, J=7.7 \mathrm{~Hz}, \mathrm{CH}_{3}\right), 5.7\left(\mathrm{t}, 2 \mathrm{H}, J=8.9 \mathrm{~Hz}, \mathrm{CH}_{3}\right), 7.6(\mathrm{~d}, 1 \mathrm{H}$, $J=7.6 \mathrm{~Hz}, \mathrm{Ar}-\mathrm{H}), 7.14(\mathrm{~d}, 1 \mathrm{H}, J=7.5 \mathrm{~Hz}, \mathrm{Ar}-\mathrm{H}), 7.21(\mathrm{dd}, 1 \mathrm{H}, \mathrm{Ar}-\mathrm{H}), 7.5(\mathrm{~s}, 1 \mathrm{H}, \mathrm{Ar}-\mathrm{H}), 6.9(\mathrm{~d}, 1 \mathrm{H}, J=7.4$ $\mathrm{Hz}, \mathrm{Ar}-\mathrm{H}), 7.34$ (d, 1H, J=7.5 Hz, Ar-H), 7.25 (dd,1H, Ar-H), 7.9 (s, 1H, Ar-H); ${ }^{13} \mathrm{CNMR}\left(\mathrm{CDCl}_{3}\right):(\delta$, ppm):215.6 (C=O), $144.8(\mathrm{C}=\mathrm{N}), 41.5\left(\right.$ Aliph- $\left.\mathrm{CH}_{2}\right), 34.3\left(\right.$ Aliph- $\left.\mathrm{CH}_{2}\right), 40.3\left(\mathrm{CH}_{2}\right), 55.8\left(\mathrm{CH}_{2}\right)$; FAB MAS: $\mathrm{m} / z\left(\mathrm{M}^{+}+1\right) 347.76$ calc.347.24. Anal. Calc. (\%) for $\left(\mathrm{C}_{18} \mathrm{H}_{16} \mathrm{Cl}_{2} \mathrm{~N}_{2} \mathrm{O}\right) \mathrm{C}, 62.26 ; \mathrm{H}, 4.64, \mathrm{~N}$, 8.07; Found: C, 62.43; H, 6.47, N, 8.17.

\section{1-(3-bromophenyl)-3-(3-(3-bromophenyl)-4,5-dihydropyrazol-1-yl)propan-1-one (11)}

Pale green solid; Yield: 59\%; m.p. $120^{\circ} \mathrm{C}$; UV: $\lambda_{\max }(\mathrm{nm}): 298,234,214$; IR: $v_{\max } \mathrm{cm}^{-1}: 1678(\mathrm{C}=\mathrm{O}), 1574$ $(\mathrm{C}=\mathrm{N}), 1167(\mathrm{C}-\mathrm{N}), 782,819$ (Benzene); ${ }^{1} \mathrm{HNMR}\left(\mathrm{CDCl}_{3}\right):(\delta, \mathrm{ppm}): 2.73\left(\mathrm{t}, 2 \mathrm{H}, J=6.4 \mathrm{~Hz}\right.$, Aliph- $\left.\mathrm{CH}_{3}\right)$, $2.71(\mathrm{t}, 2 \mathrm{H}, J=6.5 \mathrm{~Hz} \text {, Aliph-CH})_{3}, 3.44\left(\mathrm{t}, 2 \mathrm{H}, J=7.4 \mathrm{~Hz}, \mathrm{CH}_{3}\right), 5.2\left(\mathrm{t}, 2 \mathrm{H}, J=8.6 \mathrm{~Hz}, \mathrm{CH}_{3}\right), 7.5(\mathrm{~d}, 1 \mathrm{H}$, $J=7.4 \mathrm{~Hz}, \mathrm{Ar}-\mathrm{H}), 7.15(\mathrm{~d}, 1 \mathrm{H}, J=7.4 \mathrm{~Hz}, \mathrm{Ar}-\mathrm{H}), 7.26(\mathrm{dd}, 1 \mathrm{H}, \mathrm{Ar}-\mathrm{H}), 7.9$ (s,1H, Ar-H), 7.2(d, 1H, J=7.7 $\mathrm{Hz}, \mathrm{Ar}-\mathrm{H}), 7.37$ (d, 1H, J=7.5 Hz, Ar-H), $7.22(\mathrm{dd}, 1 \mathrm{H}, \mathrm{Ar}-\mathrm{H}), 7.5(\mathrm{~s}, 1 \mathrm{H}, \mathrm{Ar}-\mathrm{H}) ;{ }^{13} \mathrm{CNMR}\left(\mathrm{CDCl}_{3}\right):(\delta$, ppm): $208.1(\mathrm{C}=\mathrm{O}), 146.2(\mathrm{C}=\mathrm{N})$, 51.3(Aliph- $\left.\mathrm{CH}_{2}\right)$, 32.3(Aliph- $\left.\mathrm{CH}_{2}\right), 45.6\left(\mathrm{CH}_{2}\right), 52.8\left(\mathrm{CH}_{2}\right)$; FAB MAS: $m / z \quad\left(\mathrm{M}^{+}+1\right) 432$ calc.433.96. Anal. Calc. $(\%)$ for $\left(\mathrm{C}_{18} \mathrm{H}_{16} \mathrm{Br}_{2} \mathrm{~N}_{2} \mathrm{O}\right) \mathrm{C}, 49.57$; $\mathrm{H}, 3.70, \mathrm{~N}, 6.42$; Found: $\mathrm{C}$, 49.44; H , 3.68, N , 6.27 .

1-(4-chlorophenyl)-3-(3-(4-chlorophenyl)-4-5-dihydropyrazol-1-yl) propan-1-one (12)

Pale green solid; Yield: 88\%; m.p. $93^{\circ} \mathrm{C}$; UV: $\lambda_{\max }(\mathrm{nm}): 294,239$, 218; IR: $v_{\max } \mathrm{cm}^{-1}: 1675(\mathrm{C}=\mathrm{O}), 1581$ $(\mathrm{C}=\mathrm{N}), 1165(\mathrm{C}-\mathrm{N}), 822,881$ (Benzene) ${ }^{1} \mathrm{HNMR}\left(\mathrm{CDCl}_{3}\right):(\delta, \mathrm{ppm}): 2.73$ (t, 2H , J=6.7, Aliph-CH$\left.{ }_{3}\right)$, $2.71\left(\mathrm{t}, 2 \mathrm{H}, J=6.4\right.$, Aliph- $\left.\mathrm{CH}_{3}\right), 3.16\left(\mathrm{t}, 2 \mathrm{H}, J=6.6 \mathrm{~Hz}, \mathrm{CH}_{3}\right), 4.67\left(\mathrm{t}, 2 \mathrm{H}, J=7.5 \mathrm{~Hz}, \mathrm{CH}_{3}\right), 7.31(\mathrm{~d}, 2 \mathrm{H}$, $J=7.3 \mathrm{~Hz}, \mathrm{Ar}-\mathrm{H}), 7.19$ (d, 2H, J=7.3 Hz, Ar-H),7.19 (d, 2H, J=7.5 Hz, Ar-H), 7.41 (d, 2H, J=7.5 Hz, Ar$\mathrm{H}),{ }^{13} \mathrm{CNMR}\left(\mathrm{CDCl}_{3}\right):(\delta, \mathrm{ppm}): 235.1(\mathrm{C}=\mathrm{O}), 142.2(\mathrm{C}=\mathrm{N}), 57.3\left(\right.$ Aliph- $\left.\mathrm{CH}_{2}\right), 35.3\left(\right.$ Aliph- $\left.\mathrm{CH}_{2}\right), 58.6$ 
$\left(\mathrm{CH}_{2}\right), 67.8\left(\mathrm{CH}_{2}\right)$; FAB MAS: $m / z\left(\mathrm{M}^{+}+1\right) 345.3$ calc.346.06. Anal. Calc. (\%) for $\left(\mathrm{C}_{18} \mathrm{H}_{16} \mathrm{Cl}_{2} \mathrm{~N}_{2} \mathrm{O}\right) \mathrm{C}$, 62.26; H, 4.64, N,8.07; Found: C, 62.41; H, 4.68, N, 8.12.

3-(4,5-dihydro-4-methyl-3-phenylpyrazol-1-yl)-1-phenylpropan-1-one (17)

Pale yellow solid; Yield: 52\%; m.p.85 ${ }^{\circ} \mathrm{C}$; UV: $\lambda_{\max }(\mathrm{nm}): 288,221,236$; IR: $v_{\max } \mathrm{cm}^{-1}: 1634$ ( C=O), 1573 $(\mathrm{C}=\mathrm{N}), 1155(\mathrm{C}-\mathrm{N}), 741,687$ (Benzene); ${ }^{1} \mathrm{HNMR}\left(\mathrm{CDCl}_{3}\right):(\delta, \mathrm{ppm}): 2.76\left(\mathrm{t}, 2 \mathrm{H}, \mathrm{J}=6.5 \mathrm{~Hz}, \mathrm{Aliph}-\mathrm{CH}_{3}\right)$, $\left.2.62(\mathrm{t}, 2 \mathrm{H}, \mathrm{J}=6.7 \mathrm{~Hz}, \text { Aliph-CH})_{3}\right), 3.39\left(\mathrm{t}, 1 \mathrm{H}_{\mathrm{a}}, J=4.1 \mathrm{~Hz}, \mathrm{CH}\right), 3.87\left(\mathrm{dd}, 1 \mathrm{H}_{\mathrm{b}}, \mathrm{J}=4.1 \mathrm{~Hz}, 11.3 \mathrm{~Hz}, \mathrm{CH}\right),(3.61$

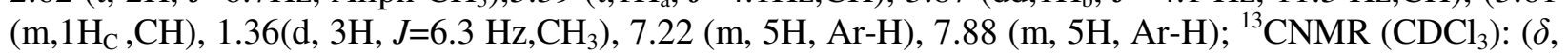
ppm): $225.2(\mathrm{C}=\mathrm{O}), 159.3(\mathrm{C}=\mathrm{N})$, 53.3 $\left(\right.$ Aliph- $\left.\mathrm{CH}_{2}\right), 42.3\left(\right.$ Aliph- $\left.\mathrm{CH}_{2}\right), 38.3(\mathrm{CH}), 56.1\left(\mathrm{CH}_{2}\right), 13.8\left(\mathrm{CH}_{3}\right)$; FAB MAS: $m / z\left(\mathrm{M}^{+}+1\right) 294$ calc.292.16. Anal. Calc. $(\%)$ for $\left(\mathrm{C}_{19} \mathrm{H}_{20} \mathrm{~N}_{2} \mathrm{O}\right): \mathrm{C}, 78.05 ; \mathrm{H}, 6.89, \mathrm{~N}, 9.58$; Found: C, 78.22; H, 6.84, N , 9.41.

\section{1-(3-chlorophenyl)-3-(3-(3-chlorophenyl)-4,5-dihydro-4-methylpyrazol-1-yl)propan-1-one (18)}

Pale yellow solid;Yield: 57\%; m.p. $95{ }^{\circ} \mathrm{C}$; UV: $\lambda_{\max }(\mathrm{nm}): 287,213,241 ; \mathrm{IR}: v_{\max } \mathrm{cm}^{-1}: 1644$ ( C=O),1521 $(\mathrm{C}=\mathrm{N}), 1162(\mathrm{C}-\mathrm{N}), 789,796$ (Benzene); ${ }^{1} \mathrm{HNMR}\left(\mathrm{CDCl}_{3}\right):(\delta, \mathrm{ppm}): 2.57$ ( t, $2 \mathrm{H}, J=6.1 \mathrm{~Hz}$, Aliph-CH$\left.{ }_{3}\right)$ , $2.74\left(\mathrm{t}, 2 \mathrm{H}, J=6.6 \mathrm{~Hz}\right.$, Aliph- $\left.\mathrm{CH}_{3}\right), 3.45\left(\mathrm{t}, 1 \mathrm{H}_{\mathrm{a}}, J=4.6 \mathrm{~Hz}, \mathrm{CH}\right), 3.86\left(\mathrm{dd}, 1 \mathrm{H}_{\mathrm{b}}, \mathrm{J}=4.6 \mathrm{~Hz}, 10.1 \mathrm{~Hz}, \mathrm{CH}\right)$, (3.61 (m,1H $\left.\mathrm{H}_{\mathrm{C}} \mathrm{CH}\right), 1.36\left(\mathrm{~d}, 3 \mathrm{H}, J=6.4 \mathrm{~Hz}, \mathrm{CH}_{3}\right), 7.2(\mathrm{~d}, 1 \mathrm{H}, J=7.2 \mathrm{~Hz}, \mathrm{Ar}-\mathrm{H}), 7.21(\mathrm{~d}, 1 \mathrm{H}, J=7.3 \mathrm{~Hz}, \mathrm{Ar}-\mathrm{H})$, $7.33(\mathrm{dd}, 1 \mathrm{H}, \mathrm{Ar}-\mathrm{H}), 7.2(\mathrm{~s}, 1 \mathrm{H}, \mathrm{Ar}-\mathrm{H}), 7.1(\mathrm{~d}, 1 \mathrm{H}, J=7.2 \mathrm{~Hz}, \mathrm{Ar}-\mathrm{H}), 7.38(\mathrm{~d}, 1 \mathrm{H}, J=7.3 \mathrm{~Hz}, \mathrm{Ar}-\mathrm{H}), 7.31$ $(\mathrm{dd}, 1 \mathrm{H}, \mathrm{Ar}-\mathrm{H}), 7.13(\mathrm{~s}, 1 \mathrm{H}, \mathrm{Ar}-\mathrm{H}) ;{ }^{13} \mathrm{CNMR}\left(\mathrm{CDCl}_{3}\right)$ : $(\delta, \mathrm{ppm}): 223.6(\mathrm{C}=\mathrm{O}), 163.3(\mathrm{C}=\mathrm{N}), 56.9$ (Aliph$\left.\mathrm{CH}_{2}\right)$, 46.3 (Aliph- $\left.\mathrm{CH}_{2}\right)$, $41.4(\mathrm{CH}), 55.3\left(\mathrm{CH}_{2}\right), 16.8\left(\mathrm{CH}_{3}\right)$; FAB MAS: $m / z\left(\mathrm{M}^{+}+1\right) 360.4$ calc. 360.08.Anal. Calc. (\%) for $\left(\mathrm{C}_{19} \mathrm{H}_{18} \mathrm{Cl}_{2} \mathrm{~N}_{2} \mathrm{O}\right)$ : C, 63.17; H, 5.02, N,7.75; Found: $\mathrm{C}, 63.14 ; \mathrm{H}, 5.21, \mathrm{~N}$, 7.65 .

\section{1-(3-bromophenyl)-3-(3-(3-bromophenyl)-4,5-dihydro-4-methylpyrazol-1-yl)propan-1-one (19)}

Pale yellow solid; Yield: $47 \%$; m.p. $88^{\circ}$ C. UV: $\lambda_{\max }(\mathrm{nm}): 299,223$, 239; IR: $v_{\max } \mathrm{cm}^{-1}: 1647$ ( C=O), 1554 $(\mathrm{C}=\mathrm{N}), 1169(\mathrm{C}-\mathrm{N}), 788,776$ (Benzene); ${ }^{1} \mathrm{HNMR}\left(\mathrm{CDCl}_{3}\right):(\delta, \mathrm{ppm}): 2.51$ ( t, 2H, J=6.4 Hz, Aliph-CH$)$, $2.86\left(\mathrm{t}, 2 \mathrm{H}, J=6.2 \mathrm{~Hz}\right.$, Aliph- $\left.\mathrm{CH}_{3}\right), 3.37\left(\mathrm{t}, 1 \mathrm{H}_{\mathrm{a}}, \mathrm{J}=4.6 \mathrm{~Hz}, \mathrm{CH}\right), 3.75\left(\mathrm{dd}, 1 \mathrm{H}_{\mathrm{b}}, \mathrm{J}=4.6 \mathrm{~Hz}, 10.1 \mathrm{~Hz}, \mathrm{CH}\right)$, $3.64\left(\mathrm{~m}, 1 \mathrm{H}_{\mathrm{C}}, \mathrm{CH}\right), 1.35\left(\mathrm{~d}, 3 \mathrm{H}, \mathrm{J}=6.4 \mathrm{~Hz}, \mathrm{CH}_{3}\right), 7.6(\mathrm{~d}, 1 \mathrm{H}, \mathrm{J}=7.2 \mathrm{~Hz}, \mathrm{Ar}-\mathrm{H}), 7.23(\mathrm{~d}, 1 \mathrm{H}, J=7.3 \mathrm{~Hz}, \mathrm{Ar}-$ H), $7.46(\mathrm{dd}, 1 \mathrm{H}, \mathrm{Ar}-\mathrm{H}), 7.2(\mathrm{~s}, 1 \mathrm{H}, \mathrm{Ar}-\mathrm{H}), 7.31(\mathrm{~d}, 1 \mathrm{H}, J=7.2 \mathrm{~Hz}, \mathrm{Ar}-\mathrm{H}), 7.35(\mathrm{~d}, 1 \mathrm{H}, J=7.3 \mathrm{~Hz}, \mathrm{Ar}-\mathrm{H})$, $7.36(\mathrm{dd}, 1 \mathrm{H}, \mathrm{Ar}-\mathrm{H}), 7.12(\mathrm{~s}, 1 \mathrm{H}, \mathrm{Ar}-\mathrm{H}) ;{ }^{13} \mathrm{CNMR}\left(\mathrm{CDCl}_{3}\right):(\delta, \mathrm{ppm}): 45.2(\mathrm{C}=\mathrm{O}), 164.3(\mathrm{C}=\mathrm{N}), 63.9$ (Aliph-CH ${ }_{2}$ ), $43.3\left(\right.$ Aliph- $\left.\mathrm{CH}_{2}\right), 45.3(\mathrm{CH}), 54.7\left(\mathrm{CH}_{2}\right), 16.3\left(\mathrm{CH}_{3}\right)$; FAB MAS: $m / z\left(\mathrm{M}^{+}+1\right) 448.3$ calc. 447.98. Anal. Calc. (\%) for $\left(\mathrm{C}_{19} \mathrm{H}_{18} \mathrm{Br}_{2} \mathrm{~N}_{2} \mathrm{O}\right)$ : C, 50.69; H, 4.23, N, 6.22 ; Found: C, 50.56; $\mathrm{H}, 4.2, \mathrm{~N}$, 6.14

\section{Synthesis of 3-Furyl-2-Pyrazoline (13)}

A $(0.1 \mathrm{~mol})$ of mannich base precursor in $70 \mathrm{ml}$ of methanol was added in $14 \mathrm{ml}$ of hydrazine hydrate, 7.2 $\mathrm{ml}$ of $50 \%$ sodium hydroxide, and $18 \mathrm{ml}$ of methanol followed by refluxing for $45 \mathrm{~min}$. The methanol was distilled off after refluxing, extract it with dichloromethane and water. Dried in vacuo gave the pure 3-furyl-2-pyrazoline which could be used without further purification.

\section{3-Furyl-2-Pyrazoline (13)}

Yellow solid, Yield: $57 \%$, m.p.: $45^{\circ} \mathrm{C}$; UV(DMSO) : 210, 315; IR: $v_{\max } \mathrm{cm}^{-1}: 3126 v(\mathrm{~N}-\mathrm{H}), 1550 v(\mathrm{C}=\mathrm{N})$, $1247 v(\mathrm{C}-\mathrm{O}-\mathrm{C}) ;{ }^{1} \mathrm{H}$ NMR (DMSO): $(\delta, \mathrm{ppm}): 7.24$ (s, H,NH); 7.97(d, H, furan H), 7.17(dd, H, furan H), 6.54(d, H, furan H); ${ }^{13} \mathrm{CNMR}(\mathrm{DMSO})$ : $(\delta, \mathrm{ppm})$ : $152.63(\mathrm{C}=\mathrm{N}), 148.33,146.50,143.44,117.55$, (ArC).Anal. Calc. (\%) for $\left(\mathrm{C}_{7} \mathrm{H}_{8} \mathrm{~N}_{2} \mathrm{O}\right)$ : C, 61.76; H, 5.88; N, 20.58; Found: C, 61.70; H, 5.80; N,20.51.

\section{Synthesis of $\mathrm{Co}(\mathrm{II}), \mathrm{Cu}(\mathrm{II}), \mathrm{Ni}$ (II) and Fe (II) Complexes $(\mathbf{2 0 , 2 1 , 2 2 , 2 3 )}$}

The metal complexes were prepared by adding 1:2 molar ratio of salts of cobalt(II), copper(II), nickel (II) $(1 \mathrm{mmol})$ in $20 \mathrm{ml}$ of ethanol to the pyrazoline $(2 \mathrm{mmol})$ dissolved in $40 \mathrm{ml}$ of ethanol with stirring. The metal complexes of iron (II) were made by adding an equal amount of ligand and iron(II) sulphate. Reflux it for $2 \mathrm{~h}$. The resulting mixture was cooled, filtered and reduced to nearly half its volume. Upon keeping the concentrated solution overnight at room temperature. The solid product formed, filtered, washed it with ethanol followed by ether and dried. 


\section{Bis(3-furyl-2-pyrazoline) Cu(II) (20)}

Brownish green solid, Yield: 56\%; m.p: $190^{\circ} \mathrm{C}, \mathrm{UV}(\mathrm{DMSO}): 224,327,462,630, \mu_{\mathrm{eff}}(\mathrm{BM}): 1.9$, IR: $v_{\max } \mathrm{cm}^{-1}: \quad 3120(\mathrm{~N}-\mathrm{H}), \quad 1565(\mathrm{C}=\mathrm{N}), 1235(\mathrm{C}-\mathrm{O}-\mathrm{C}), \quad 308(\mathrm{M}-\mathrm{N}), \quad 441.9(\mathrm{M}-\mathrm{O}) .{ }^{1} \mathrm{H}$ NMR (DMSO): $(\delta$, ppm):7.20 (s, H, NH), $7.95(\mathrm{~d}, \mathrm{H}$, furan $\mathrm{H}) 7.15$ (dd, $\mathrm{H}$, furan $\mathrm{H}), 6.53(\mathrm{~d}, \mathrm{H}$, furan $\mathrm{H}) ;{ }^{13} \mathrm{CNMR}(\mathrm{DMSO})$ : $(\delta, \mathrm{ppm}): 153.27(\mathrm{C}=\mathrm{N}), 148.41,146.73,143.44,177.59\left(\right.$ Ar-C).Anal. Calc. $(\%)$ for $\left(\mathrm{C}_{14} \mathrm{H}_{16} \mathrm{~N}_{4} \mathrm{O}_{2} \mathrm{Cu}\right): \mathrm{C}$, 50.07; H.4.77; N, 16.69; Found: C, 50.07; H, 4.76; N, 16.6.

\section{Diaquabis(3-furyl-2-pyrazoline)Ni(II)Nitrate (21)}

Blackish red solid, Yield: $45 \%$ m.p: $264^{\circ} \mathrm{C}$, UV(DMSO): 261, 333, 440, 650; $\mu_{\text {eff }}(\mathrm{BM}): 2.95 ;$ IR: $v_{\max } \mathrm{cm}^{-}$ ${ }^{1}:$ 3112(N-H), 1590(C=N),1241(C-O-C), 306(M-N), 489v(M-O). ${ }^{1} \mathrm{H}$ NMR (DMSO): $(\delta, \mathrm{ppm}): 7.19$ (s, H, $\mathrm{NH}), 7.99(\mathrm{~d}, \mathrm{H}$, furan $\mathrm{H}), 7.12(\mathrm{dd}, \mathrm{H}$, furan $\mathrm{H}), 6.52(\mathrm{~d}, \mathrm{H}$, furan $\mathrm{H}) ;{ }^{13} \mathrm{CNMR}(\mathrm{DMSO})$ : $(\delta$, ppm): 152.96(C=N),148.33, 146.71, 143.43, 17.53(Ar-C); Anal. Calc. (\%)for $\left(\mathrm{C}_{14} \mathrm{H}_{20} \mathrm{~N}_{6} \mathrm{O}_{9} \mathrm{Ni}\right)$ : C,34.28; H.4..08; N,17.14; Found:C, 34.28; H, 4.01; N, 17.1.

\section{Diaquasulphato(3-furyl-2-pyrazoline)iron(II) (22)}

Dark red solid, Yield: 50\%. m.p.: $290^{\circ} \mathrm{C}$, UV(DMSO): 236, 321, 370, 659; $\mu_{\mathrm{eff}}(\mathrm{BM}): 4.9 ;$ IR: $v_{\max } \mathrm{cm}^{-1}$ : 3107(N-H), 1565(C=N),1205(C-O-C), 304(M-N), 537(M-O). ${ }^{1} \mathrm{H}$ NMR (DMSO): $(\delta, \mathrm{ppm}): 7.26$ (s, H, $\mathrm{NH}), 7.93(\mathrm{~d}, \mathrm{H}$, furan $\mathrm{H}) 7.14(\mathrm{dd}, \mathrm{H}$, furan $\mathrm{H}), 6.54(\mathrm{~d}, \mathrm{H}$, furan $\mathrm{H}) ;{ }^{13} \mathrm{CNMR}(\mathrm{DMSO}):(\delta$, ppm): 153.13(C=N),148.53, 146.67, 143.40, 117.51(Ar-C).Anal. Calc.(\%) for $\left(\mathrm{C}_{7} \mathrm{H}_{8} \mathrm{~N}_{2} \mathrm{O}_{7} \mathrm{FeS}\right)$ : $\mathrm{C}, 25.92 ; \mathrm{H}, 2.46$; N,8.64; Found: C, 25.92; H, 2.37; N, 8.6.

Dichlorobis(3-furyl-2-pyrazoline)cobalt(II) (23)

Brown solid, Yield: 68\%. m.p.: $200{ }^{\circ} \mathrm{C}$, UV(DMSO): 244, 347, 460, 550; $\mu_{\mathrm{eff}}(\mathrm{BM}): 4.67$ IR: $v_{\max } \mathrm{cm}^{-1}$ : 3111(N-H), $1579(\mathrm{C}=\mathrm{N}), 1233(\mathrm{C}-\mathrm{O}-\mathrm{C}), 323(\mathrm{M}-\mathrm{N}), 502(\mathrm{M}-\mathrm{O}) .{ }^{1} \mathrm{H}$ NMR (DMSO): $(\delta, \mathrm{ppm}): 7.25$ (s, H, $\mathrm{NH}), 7.96(\mathrm{~d}, \mathrm{H}$, furan $\mathrm{H}) 7.14(\mathrm{dd}, \mathrm{H}$, furan $\mathrm{H}), 6.55(\mathrm{~d}, \mathrm{H}$, furan $\mathrm{H}) ;{ }^{13} \mathrm{CNMR}(\mathrm{DMSO}):(\delta, \mathrm{ppm})$ : 52.71(C=N), 148.39, 146.48, 143.41, 117.57(Ar-C).Anal. Calc.(\%)for $\left.\mathrm{C}_{14} \mathrm{H}_{16} \mathrm{~N}_{4} \mathrm{O}_{2} \mathrm{Cl}_{2} \mathrm{Co}\right): \mathrm{C}, 41.80 ; \mathrm{H}$, 3.98; N,13.33; Found:C, 41.80; H, 3.91; N, 13.19 .

\section{Synthesis of Ru(II) and Pd(II) complexes(24,26)}

To a solution of $\mathrm{RuCl}_{3} .3 \mathrm{H} 2 \mathrm{O}$ (1 $\left.\mathrm{mmol}\right)$ in dry Methanol $(20 \mathrm{ml})$ under argon was added ligand $(1 \mathrm{mmol})$ in methanol $(10 \mathrm{ml})$, then add $\mathrm{Zn}$ powder $(0.316 \mathrm{~g}, 4.8 \mathrm{mmol})$. Refluxed it for $8 \mathrm{~h}$. The product was filtered and con to half of its volume under a stream of argon. Anhy. Diethyl ether was added, until the solution became turbid. A brown solid deposited, filtered, washed with methanol and diethyl ether.

\section{Dichlorobis(3-furyl-2-pyrazoline)palladium(II) (24)}

Brown solid, Yield: $71 \%$. m.p.: $175^{\circ} \mathrm{C}$, UV(DMSO): 218, 338, 365,394, 407; IR: $v_{\max } \mathrm{cm}^{-1}: 3123$ (N-H), $1576(\mathrm{C}=\mathrm{N}), 1205$ (C-O-C), 515(M-N), 475 (M-S); ${ }^{1} \mathrm{H}$ NMR (DMSO): ( $\delta$, ppm): 7.26 (s, H, NH); 7.93 (d, $\mathrm{H}$, furan $\mathrm{H}) 7.19(\mathrm{dd}, \mathrm{H}$, furan $\mathrm{H}), 6.49(\mathrm{~d}, \mathrm{H}$, furan $\mathrm{H}) ;{ }^{13} \mathrm{CNMR}(\mathrm{DMSO})$ : $(\delta$, ppm): 155.21(C=N),148.61, 146.79, 143.46, 117.59 (Ar-C ).Anal. Calc.(\%)for $\left(\mathrm{C}_{7} \mathrm{H}_{8} \mathrm{~N}_{2} \mathrm{OPdCl}{ }_{2}\right): \mathrm{C}, 26.83$; H.2.55; N, 8.94; Found: C, 26.8; H, 2.5; N, 8.9.

\section{Diaquadichloro(3-furyl-2-pyrazoline)ruthenium(II) (26)}

Dark brown solid, Yield: $45 \%$., m.p. : $177^{\circ} \mathrm{C}$, UV(DMSO): 217, 327, 614, 410, IR: $v_{\max } \mathrm{cm}^{-1}: 3126 v(\mathrm{~N}-\mathrm{H})$, $1569 v(\mathrm{C}=\mathrm{N}), 1233 v(\mathrm{C}-\mathrm{O}-\mathrm{C}), 525(\mathrm{M}-\mathrm{N}), 490(\mathrm{M}-\mathrm{O}) ;{ }^{1} \mathrm{H}$ NMR (DMSO): $(\delta, \mathrm{ppm}): 7.17(\mathrm{~s}, \mathrm{H}, \mathrm{NH})$; $7.92(\mathrm{~d}, \mathrm{H}$, furan $\mathrm{H}), 7.14(\mathrm{dd}, \mathrm{H}$, furan $\mathrm{H}), 6.50(\mathrm{~d}, \mathrm{H}$, furan $\mathrm{H}) ;{ }^{13} \mathrm{CNMR}(\mathrm{DMSO}):(\delta$, ppm): 153.41 $(\mathrm{C}=\mathrm{N}), 148.33,146.54,143.47,117.55(\mathrm{Ar}-\mathrm{C})$. Anal. Calc. (\%)for $\left(\mathrm{C}_{7} \mathrm{H}_{12} \mathrm{~N}_{2} \mathrm{O}_{3} \mathrm{NRuCl}_{2}\right)$ : $\mathrm{C}, 24.41$; H, 3.48; N, 8.13; Found: C, 24.4; H, 3.41; N, 8.1.

\section{Synthesis of Pd (II) and Pt(II) complexes $(25,27)$}

All Pd (II) and Pt(II) complexes were prepared by adding an equal amount of both ligands and metal precursor, we use dry methanol as a solvent, reflux it about $4 \mathrm{~h}$. Leave it overnight at $0^{\circ} \mathrm{C}$. The precipitate formed, filtered, washed the product and dried it.

\section{Dichlorobis(3-furyl-2-pyrazoline)platinum(II) (25)}

Brown solid, Yield: $80 \%$. m.p : $152^{\circ} \mathrm{C}$; UV(DMSO): 224, 320, 345, 356, 417; IR: $v_{\max } \mathrm{cm}^{-1}: 3119$ (N-H), 1565(C=N), 1240(C-O-C), 518(M-N), 439 v(M-O); ${ }^{1} \mathrm{H}$ NMR (DMSO): ( $\delta$, ppm): 7.21 (s, H, NH); 7.95 
$(\mathrm{d}, \mathrm{H}$, furan $\mathrm{H}), 7.13$ (dd, $\mathrm{H}$, furan $\mathrm{H}), 6.57$ (d, $\mathrm{H}$, furan $\mathrm{H}) ;{ }^{13} \mathrm{CNMR}(\mathrm{DMSO})$ : $(\delta$, ppm): 152.97(C=N),149.01, 146.50, 143.75, 117.55 (Ar-C).Anal. Calc. (\%)for $\left(\mathrm{C}_{7} \mathrm{H}_{8} \mathrm{~N}_{2} \mathrm{OPtCl}{ }_{2}\right)$ : C, 20.89 ; H.1.99; N, 6.96; Found:C, 20.6; H, 1.9; N, 6.9.

\section{Diacetato(3-furyl-2-pyrazoline)palladium(II) (27)}

Yellowish orange solid, Yield : $51 \%$, m.p. : $172^{\circ} \mathrm{C}$; UV(DMSO): 227, 325, 355, 367, 415; IR: $v_{\max } \mathrm{cm}^{-1}$ : $3089(\mathrm{~N}-\mathrm{H}), 1540(\mathrm{C}=\mathrm{N}), 869(\mathrm{C}-\mathrm{S}-\mathrm{C}), 540(\mathrm{M}-\mathrm{N}), 352(\mathrm{M}-\mathrm{N}) ;{ }^{1} \mathrm{H}$ NMR (DMSO): $(\delta, \mathrm{ppm}): 7.29$ (s, H, $\mathrm{NH}) ; 7.91(\mathrm{~d}, \mathrm{H}$, furan $\mathrm{H}), 7.19(\mathrm{dd}, \mathrm{H}$, furan $\mathrm{H}), 6.54(\mathrm{~d}, \mathrm{H}$, furan $\mathrm{H}) ;{ }^{13} \mathrm{CNMR}(\mathrm{DMSO})$ : $(\delta$, ppm): 152.87(C=N), 148.5, 146.50, 143.44, 117.55(Ar-C).Anal. Calc.(\%)for $\left(\mathrm{C}_{11} \mathrm{H}_{14} \mathrm{~N}_{2} \mathrm{O}_{5} \mathrm{Pd}\right)$ : $\mathrm{C}, 36.66 ; \mathrm{H}, 3.88$; N,7.77; Found: C, 3.66; H, 3.8; N, 7.72

\section{Antiamoebic Activity}

The compounds were screened in vitro for antiamoebic activity against the HM1:IMSS strain of $E$. histolytica by the microdilution method. ${ }^{38}$ E. histolytica trophozoites were cultured in wells of 96 microtiter plate (Costar) in TYIS-33 growth medium. ${ }^{39}$ In DMSO compounds were dissolved at which level amoeba occurs showed no inhibition. ${ }^{40} 2$ fold dilutions were used. We use $1 \mathrm{mg} / \mathrm{ml}$ of concentration of culture medium. Metronidazole used as a standard drug. To confirm viability trypan blue exclusion used. By adding fresh medium of dilution $10^{5}$ organisms $/ \mathrm{ml}$ in cell suspension and $170 \mu \mathrm{l}$ of this suspension was used in test and control wells plate. We chose inoculum of $1.7 \times 10^{4}$ organisms/well, after that growth took place in it. The plates were sealed, and gassed for $10 \mathrm{~min}$ in a Nitrogen, and incubated was performed at $37^{\circ} \mathrm{C}$ for 72 hours. With microscope, we checked the growth of the amoeba. By inverting and shaking the plate culture medium was removed. Then plates were washed with $0.9 \%$ aq. $\mathrm{NaCl}$ solution. To prevent the detachment of amoebae, the plate was not allowed to cool. The amoeba was fixed with chilled methanol in an ice bath for $15 \mathrm{~min}$. dried and stained with $0.5 \%$ aq. Eosin for 15 min. After that stained plate was washed with distilled water and then dries it. To dissolved protein $0.1 \mathrm{~N}$ aq. $\mathrm{NaOH}$ solution was added to each well. From the optical density of the control and test wells, we calculate inhibition (\%) of amoebal growth. From the Linear-regression analysis, we calculate $\mathrm{IC}_{50}$ values. The experiments were performed thrice for each compound.

\section{In silico Physic-Chemical Properties}

The evaluation of important physio-chemical properties of drugs like molecules is an important step in the process of drug discovery. The ADMET parameter for all the nineteen compounds (1-19) was evaluated using pkCSM online webserver (http://biosig.unimelb.edu.au/pkcsm) and carcinogenic properties were determined CarcinoPred-EL webserver. Different important description such as molecular weight (MW), Dipole Moment (D), total polar surface area (PSA), number of HBD, blood-brain partition coefficient ( $\log \mathrm{Kp}$ ), Lipinski rule of 5 violations, percentage human oral absorption, lipophilicity parameter [log $\mathrm{P}(\mathrm{o} / \mathrm{w})$ ], solubility $(\log \mathrm{S})$ with many others were calculated. According to Lipinski rule of five, any orally active drugs should not violate more than one of its parameters. The important Lipinski parameters are the number of HBA (not more than 10), number of HBD (not more than 5), molecular mass (less than 500), partition coefficient $(\log p \leq 5)$ and molar refractivity (in the range of 40-130). The ideal range of these descriptors was based on the reference of $95 \%$ drugs.

\section{Docking Studies}

Molecular docking studies were performed to determine the interaction between lead compounds and possible drug targets. Thioredoxin reductase of E.histolityca (EhTrR) (PDB ID: 4a65) was selected for docking purposess. Complex crystal structure of the protein got from RCSB protein data bank (https://www.rcsb.org/pdb) and processed before docking. The structures of ligands were drawn using ChemSketch which were further converted into PDB format by online SMILES translator. The energy minimization of ligands and protein was done by Swiss PDB Viewer and converted into ADT desired format PDBQT using ADT tools. AutodockVina 4.2 software was used to perform docking. ${ }^{41}$ Water molecule removed in a protein processing and hydrogen added and ligand was processed for ADT format. The X, Y and Z coordinates of grid box were 56, 54 and 54, respectively with $1 \AA$ spacing which covers complete protein for the purpose of blind docking. The $\mathrm{x}, \mathrm{y}$, and $\mathrm{z}$ center coordinates were measured as 7.24, -14.093 and -13.987 , respectively. For further interface analysis complex with the minimum 
binding energy and more interactions were chosen. The visualization of protein-ligand interaction was done by PyMol. ${ }^{42}$

\section{CONCLUSION}

A series of pyrazoline derivatives and metal complexes of 3-furyl-2-pyrazoline were synthesized. We examined the antiamoebic activity of all the synthesized compounds. $\mathrm{IC}_{50}$ value suggested that the metal complexes showed better antiamoebic potential. The in silico prediction of physicochemical properties for the compounds (1-19) were found to be within the permitted range. From the molecular docking, we found that compounds 11, 12 and $\mathbf{2 0}$ are binding with thioredoxin reductase of E. histolytica $(\mathrm{EhTrR})$ as their possible way of action.

\section{ACKNOWLEDGMENT}

Iram Irfan is thanks to Prof. A Bhattacharya, School of Life Science, JNU, New Delhi, for allowing using their Laboratory.

\section{REFERENCES}

1. J. D. Ospina-Villa, N. Guillen, C. L. Camarillo, J. S. Sanchez, E. R. Moreno and E. R. Moreno, E. R. Garcia-Vazquez, C. A. Castañon-Sanchez, A. Betanzos, L. A. Marchat, Journal of Microbiology, 55, 783(2017), DOI: 10.1007/s12275-017-7259-9

2. A. A. Kelsoa, S. D. Goodsona, S. Chavan, A. F. Saya, A. Turchickand D. Sharma, L. L. Ledforda, E. Rattermana, K. Leskoskea, A. V. King, C. C. Attaway, Y. Bandera, H. Foulger, A. V. Mazin, L.V. Temesvari and M. G. Sehorna, Molecular and Biochemical Parasitolog, 210, 71(2016), DOI:10.1016/j.molbiopara.2016.09.001

3. P. Johnson, Parasitol Today, 9, 183(1993), DOI:10.1016/0169-4758(93)90143-4

4. V. Purohit and A. K. Basu, Chemical Research in Toxicology, 13, 673(2000), DOI: 10.1021/tx000002x

5. T. Cavas and S. Ergene-Gozukara, Environmental Toxicology and Pharmacology, 19, 107 (2005), DOI: $10.1016 /$ j.etap.2004.05.007

6. A. F. El-Nahas and I. M. El-Ashmawy, Basic and Clinical Pharmacology Toxicology, 94, 226(2004), DOI: $10.1111 / \mathrm{j} .1742-7843.2004 . p t o 940505 . \mathrm{x}$

7. S. Toumi, M. Hammouda, A. Essid, L. Medimagh, L.B. Slamia and C. Laouani-Kechrid, Medicine et Maladies Infectieuses, 39, 906(2008), DOI: 10.1016/j.medmal.2008.11.007

8. S. Becker, P. Hoffman and E.R. Houpt, The American Journal of Tropical Medicine and Hygiene, 84, 581(2011), DOI: 10.4269/ajtmh.2011.10-0580

9. S.M. Siddiqui, A. Salahuddin and A. Azam, European Journal of Medicinal Chemistry, 49, 411(2012), DOI: 10.1016/j.ejmech.2012.01.030

10. A. Solankee and Y. Prajapati, Rasayan Journal of Chemistry, 2 (1), 23(2009).

11. J. Rajora and Y. K. Srivastava, Rasayan Journal of Chemistry, 2 (3), 641(2009).

12. Y. K. Srivastava, Rasayan Journal of Chemistry, 1 (4), 837(2008).

13. M. SirajulMuneera, J. Joseph,Design, Journal of Photochemistry and Photobiology B: Biology, 163, 57(2016), DOI: 10.1016/j.jphotobiol.2016.08.010

14. H. Parveen, S. Mukhtar and A. Azam, Journal of Heterocyclic Chemistry, 52(2), (2015), DOI:10.1002/jhet.2427

15. M. Abid, A. R. Bhat, F. Ather and A. Azam, European Journal Medicinal Chemistry, 44, 417(2007), DOI:10.1016/j.ejmech.2007.10.032

16. K.Husain , M. Abid and A. Azam , European Journal of Medicinal Chemistry, 43(2), 393(2008), DOI: $10.1016 /$ j.ejmech.2007.03.021

17. A. Budakoti, M. Abid and A. Azam , European Journal of Medicinal Chemistry, 42, 544(2007), DOI:10.1016/j.ejmech.2006.10.011

18. A. Debnath, D. Parsonage, R.M. Andrade, E. R. Cobo, K. Hirata and S. Chem, Nature Medicine, 18, 956(2012), DOI: $10.1038 / \mathrm{nm} .2758$

19. B. Negi, K. K. Raj, S. M. Siddiqui, D. Ramachandran, Amir Azam and D. S. Rawat, Chem. Med. Chem, 9, 2343(2014), DOI:10.1002/cmdc.201402240

20. K. Wellinga, A. C. Grosscurt and R. V. Hes, Journal of Agricultural and Food Chemistry, 25 (5), 987(1977), DOI:10.1021/jf60213a018 
21. X. Du, C. Gue, E, Hansell, P.S. Doyle, C.R. Caffrey and T.P. Holler, Journal of Medicinal Chemistry, 45, 2695(2002), DOI:10.1021/jm010459j

22. M. Stamper, B. F. Avcock, Journal of American Chemistry Society,76, 2786(1953), DOI: 10.1021/ja01639a054

23. Adams, D. M. Metal-ligand and Related vibrations: A Critical Survey of the Infrared and Raman Spectra of Metallic and Organometallic Compounds, 1st E. Arnold, London, 1967.

24. Ferraro, J. R. Low-Frequency Vibrations of Inorganic and Coordination Compounds, Plenum Press, New-York, 12, 1971.

25. D. M. Adams, Metal ligands and Related Vibrations, Edward Arnold, London, 1967.

26. F.A. Cotton, G. Wilkinson, C.A. Murillo and M. Bochmann, Advanced Inorganic Chemistry, sixth ed., Wiley, New York, 1999.

27. Y. Nishida and S. Kida, Coordination Chemistry Reviews, 27, 275(1979), DOI: org/10.1016/S00108545(00)82069-X

28. C. Sharaby, Inorg. Metal Organic and Nano-Metal Chemisstry., 35, 133(2005), DOI:10.1081/SIM200035687

29. M. M. Omar and G. G. Mohamed, Spectrochimica Acta Part A, 61, 929(2005), DOI: 10.1016/j.saa.2004.05.040

30. A. A. El-Asmy, M. A. Hafez, E. M. Saad and E.I. Taha, Transition Metal Chemistry, 19,603(1994), DOI:10.1007/BF00980412

31. D. K. Demertzi, A. Domopoulou, G. Valle, M. A. Demertzis and A. Papageorgiou, Journal of Inorganic Biochemistry, 68 , 147(1997), DOI:10.1016/S0162-0134(97)00087-1

32. D. K. Demertzi, A. Domopoulou and M. A. Demertzis, Polyhedron, 16, 3625(1997), DOI: 10.1016/S0277-5387(97)00107-1

33. D. K. Demertzi, P. N. Yadav, M. A. Demertzis and M. Coluccia, Journal of Inorganic Biochemistry, 78, 347(2000), DOI:10.1016/S0162-0134(00)00063-5

34. V. Chauhan and S.K. Dikshit, Inorganic and Metal-Organic Chemistry 9, 1037(1989), DOI:.org/10.1080/00945718908048114

35. B. S. Holla, P. M. Akberali and M. K. Shivananda, Farmaco, 256(2000), DOI:10.1016/S0014$827 \mathrm{X}(00) 00030-6$

36. Andrade, M. Rosa and S. L. Reed, Frontiers in Microbiology, (6) 975, 1(2015), DOI: $10.3389 /$ fmicb.2015.00975

37. D. Parsonage, F. Sheng, K. Hirata, A. Debnath and J. H. McKerrow, Journal of Structural Biology, 194, 80(2016), DOI:10.1016/j.jsb.2016.02.015

38. W. C. Wright, M. J. O'Neill, J. D. Phillipson and D. C. Warhurst, Antimicrobial Agents and Chemotherapy, 32, 1725(1988), DOI: 10.1128/AAC.32.11.1725

39. L. S. Diamond, D. R. Harlow and C. C. Cunnick, Transaction of the Royal Society of Tropical Medicine Hygiene,72, 431(1978), DOI:10.1016/0035-9203(78)90144-X

40. F. D. Gillin, D. S. Reiner and M. Suffness, Antimicrobial Agents and Chemotherapy, 22, 342(1982), DOI: 10.1128 /AAC.22.2.342

41. Trott and Olson, AutoDockVina:, Journal of Computational Chemistry, 31, 455(2010), DOI: $10.1002 /$ jcc. 21334

42. W. DeLano, The PyMOL user's manual, DeLanoSci, San Carlos, CA, 2002.

[RJC-5320/2019] 